\title{
An Introduction into the Physics of Self-folding Thin Structures
}

\section{Introduction}

Spontaneous folding of matter has long been the subject of disparate branches of research, from the earlier vitalistic view of Leibniz ${ }^{1}$ to the mathematical description of morphogenetic processes of D'Arcy Thompson. ${ }^{2}$ Today, scientific advancements have shown how folding is a common strategy adopted in biological systems to build up more and more complex structures - in proteins, from a peptide chain to a functional enzyme; in plants, from bud petals to a developed flower; in organisms, from layers of cells to diversified embryos. One might think that such folding processes require exceptionally complex biological machineries to orchestrate them. On the contrary, with this contribution, we will show that folding can result from remarkably simple processes - and equally applies to both natural and artificial systems. In the following we will introduce the reader to the necessary theoretical concepts that are needed to understand these phenomena, providing examples from the common experience enabling a more systematic understanding.

When we talk about self-folding ${ }^{3}$ thin structures we refer to a broad class of spontaneous shape changes (that is not caused by an external load) that occur in thin bodies. The adjective thin here means that a three-dimensional body has at least one dimension much smaller than the other; that is, a rod can be considered one-dimensional since it has two small dimensions; a plate instead is two-dimensional since it has one small dimension. In

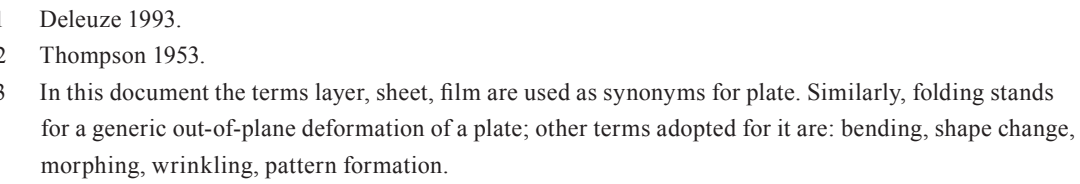


particular we will refer to bodies that can be considered two-dimensional, that is plates (which are flat) and shells (which are curved). As will become clear in section 1, the fact that plates (and shells) are thin is at the base of the rich panorama of shape changes that are observed.

The goal of the present document is to provide an overview of some of the scientific literature dealing with shape changes in self-folding thin structures. Although these shape changes can be of a very different nature, size and origin - occurring in artificial as well as biological materials, and in systems that range from nanometer to centimeter sizes - all can be understood under some unifying concepts that will be introduced in the following. Thanks to our presentation, it becomes much easier to locate where research efforts have been focused until now and to identify new fields of interest that scientific research hasn't addressed yet.

This document is organized as follows. In section 1 we introduce the reader to the basic concepts of the mechanics of a thin plate (the experienced reader can skip this part). In section 2 we will introduce many examples of self-folding systems, categorizing them on the base of how eigenstrains are distributed and orientated in the plate. In this section, we will discuss the folding (or more generally the morphing) of systems that: undergo confined expansion due to an attached elastic foundation (section 2.2); are subjected to the so-called edge growth (section 2.3); which change curvature (section 2.4). In section 3 we will give some concluding remarks on the present work.

\section{Mechanics of a thin plate: basic concepts and examples}

In this section we introduce the reader to the mechanics of a thin plate through some guiding examples. In section 1.1 we will analyze simple deformation modes like stretching and bending, introduce the concept of buckling of a thin body and also clarify why these two modes cannot be separated for curved shells. In section 1.2 we will present the concept of eigenstrain and eigencurvature and some of the different strategies to generate these in natural and artificial materials.

\subsection{Simple deformation modes of a thin body}

\subsubsection{What strains are}

To be able to discuss any self-folding system, we need to "zoom" in the material that make up these objects and describe what happens locally when such shape changes occur. As a guiding example, we take a sheet of rubber (such as a deflated balloon), mark it with a circle enclosing a small portion and conduct a series of three "handheld" experiments on 
it (fig. $1 \mathrm{a}-\mathrm{c}$ ). If we stretch it, we see that the circle becomes elongated. If we now take half of the rubber piece and stretch it by the same amount we will see that the circle is now more elongated (the elongation is now double). Stretching the sheet along a different direction will cause the circle to elongate along that direction. A useful concept to group together these intuitive results is that of strain (usually associated to the greek letter $\varepsilon$ ) in our examples the elongation of the circle with respect to its initial size. We see that the strain:

> describes the shape change of the material locally

> its magnitude can be evaluated from the ratio between the elongation and the size of the object - it is normalized

, describes the shape change along all possible directions
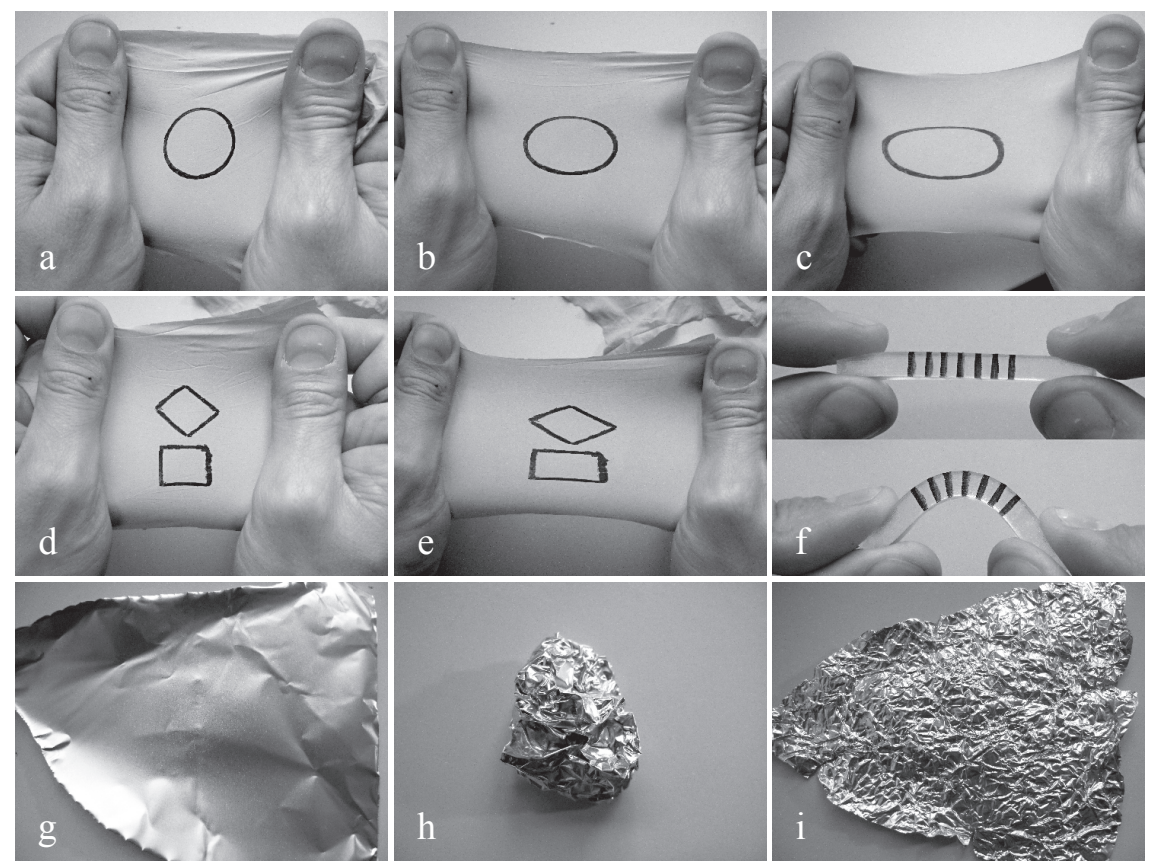

Fig. 1: Visualizing strain in a rubber sheet under increasing loading (a to c); strain depends on its frame of reference: the principal directions identify directions of pure "size-changing" strains (square to rectangle) whereas other frame orientations will give also "shape-changing" strain components (square to rhombus) (d-e); imposing a curvature to a bar generates extensional/compressive strains on its convex/concave sides (f); differently from the rubber example, an aluminum foil deformed above a small critical strain does not recover its initial shape when loads are removed $(g-i)$.

Now let us draw two squares on the sheet, close together and in the center of the sheet, one with two sides parallel to the stretching direction and the other rotated by $45^{\circ}$ degrees. After stretching the sheet one has become a rectangle, the other one a rhombus (fig. $1 \mathrm{~d}-\mathrm{e}$ ). 
It seems that the first square experiences normal strains, which measure a change in length, while the other shear strains, which quantify a pure change in shape with no change in volume: this is counterintuitive because we expect that the rubber is subjected to the same strain state (at least close to the sheet center). This evidence shows that the strains depend on the frame of reference. It also shows that in each point one can find an orientation of this frame that make the shear strains null. When this happens, the strain tensor has only components on the diagonal, and they are called principal strains.

\subsubsection{What stresses are}

The reason why the circle elongates can be made manifest by cutting the circle out of the stretched rubber: then the circle becomes round again. If we stretch two identical overlapping sheets of rubber, we will need double the force to elongate the circle by the same amount. These evidences can be unified by a quantity - the stress, usually associated to the greek letter $\sigma-$ which:

1. describes the net internal forces acting on the material locally (along all possible directions)

2. results from (and balances) the external forces of the surrounding material acting on the circle

3. is a measure of the forces acting in the material, normalized by the area of the surface on which the forces apply (in our example the area of the sheets' cross-section)

Coming back to our experiments, we notice that releasing the rubber sheet causes it to return to its initial shape, regardless of the amount of force or elongation we applied. This ability is due to the rubber's property - it is said to be elastic. Also, in the second experiment -stretching a half sheet by the same amount- we realize that the force we had to put on the rubber was, most probably, double. Recalling that also the strain doubled, it appears that the stress is proportional to the strain. The relationship between stress and strain is called the constitutive law of the material. ${ }^{4}$ This constitutive law serves to define the mechanical behavior of the material, and is a function of the material's microstructure and chemical composition. In the case of rubber and many other solid materials at low strains this relationship is linear, meaning that stress and strains are related by proportionality constant. To summarize, rubber has a linear elastic behavior (at least for not too large strains): it is also said that it follows the Hooke's law. ${ }^{5}$ Other materials (like the foil we use to wrap food, for example) would deform irreversibly when deformed above a certain strain (see fig. $1 \mathrm{~g}-\mathrm{i}$ ).

4 Callister 2007.

5 The Hooke's law describes how linear springs behave mechanically: the force needed to extend a spring increases with its extension. The proportionality factor between force and extension is the spring rigidity constant. 


\subsubsection{Bending of a plate ${ }^{6}$}

Instead of stretching the sheet, we now bend it. To highlight the strain when the sheet is bent, we draw equidistant lines on the sheet's profile perpendicularly to the sheet's long axis. At the curved site, the lines are now more closely spaced on the convex side and more widely spaced on the concave side (see fig. 1f). This means that an imposed curvature ${ }^{7}$ results in normal strains that adopt a "butterfly" distribution, negative at the convex and positive at the concave side, instead of a flat one as in the simple elongation case. Also this means that the average strains in the thickness are zero: the length of the sheet does not change, just its curvature.

When considering very thin bodies, we can make the assumption that their thickness is zero and study them as surfaces in three-dimensional space. Therefore the strain definition simplifies to only in-plane components that equal their average across the thickness. If a thin body is deformed in pure bending, under the small thickness hypothesis we would have zero energy for the bent state, because the average in-plane strain for pure bending (that is of the "butterfly" distribution) is zero. Therefore, to be able to properly describe the strain state we must define the curvature of the surface: then the imposed curvature will be associated with a strain energy. Comparing the amount of force put to bend the rubber sheet rather than stretching it, we clearly see how it is much easier to bend: the energy involved in bending is lower than in stretching, or equivalently, that the bending rigidity of a sheet is much lower than its axial rigidity. It can be shown that such mechanical behavior depends on the thin geometry of the plate: the bending energy has a prefactor $h^{3}$ and therefore will be much smaller than the stretching energy (with prefactor $h$ ) when the plate thickness $h$ is small.

\subsubsection{Buckling and stability: switching between stretching and bending modes}

To understand how shape changes occur in thin bodies it is useful to introduce a simpler example of a bar subjected to an increasing axial compression. Initially the bar shortens: at every small increment of the axial load corresponds a small increment of the bar's shortening. This fact is in accordance with the symmetry of the loading case: the bar is a one-dimensional body, the load is also directed along the bar's axis. The deformation requires work to be done on the bar: this external work is stored as strain energy in the material. In other words, conservation of energy implies that we can equate this (external) energy to an (internal) strain energy that depends from the stresses and strains. Coming back to the bar loading experiment, if the load passes a certain threshold the bar will start to bend: at this point it becomes easier to bring the ends of the bar together (lower load

6 Most of the concepts introduced in this and the next two sections have been taken from: Audoly/Pomeau 2010 .

7 For a definition of curvature refer to section 1.1.5. 
increments are required) and every increment in load will cause a large deflection from the straight axis. This phenomenon is called buckling. It shows that for a mechanical system more than one equilibrium configuration are possible ${ }^{8}$ (in our case, both the straight and slightly deflected ones are indeed possible at the buckling load threshold). In general the system will choose a deformation path which corresponds to lower energy: for a thin body (such as the bar but also a plate) this lower energy path is the bending mode. Moreover, since such buckling events are eventually caused by the different deformation modes that the material experiences, they can surface even in absence of external loading: such is the case of shape changes in the examples that will be provided in section 3, where buckling events are triggered by differential eigenstrains.

\subsubsection{Geometry of a thin body and implications for its mechanical behavior}

From a geometrical perspective, thin bodies can be considered as surfaces. To describe the shape of a surface in three dimensions, we first step back and consider, for simplicity, a line drawn on a plane. Its shape is characterized by the curvature, defined as the inverse of the radius of the circle tangent to it. For every point of the line the curvature is single-valued. If we consider a point on a generic surface instead, we can draw an infinite number of lines passing through that point. In this case each point is associated with an infinite number of curvatures depending on the orientation of the corresponding line. In reality these curvatures are related together: similarly to what we have seen for strain and stress, it suffices to know the two principal curvatures (the maximum and minimum curvatures of all possible ones), which are oriented along perpendicular directions, to fully characterize the curvature state.

Inspecting the values of the principal curvatures for different regular surfaces is instructive. Then for a plane both are clearly 0 . For a cylinder ${ }^{9}$ these are 0 and $1 / R$, respectively parallel and perpendicular to a straight line drawn on its surface. For a sphere these are both $1 / \mathrm{R}$. Defining the mean curvature $\mathrm{H}$ as the average between the principal ones, we have a way to measure the total "amount of curvature": the sphere has a higher curvature than the cylinder, which is more curved than the plane. Another fundamental measure of curvature is the Gaussian curvature $\mathrm{K}_{\mathrm{G}}$, defined as the product of the principal ones (fig. 2). The meaning of $\mathrm{K}_{\mathrm{G}}$ can be easily understood by comparing its value in the three surfaces we just presented. Plane and cylinder have same Gaussian curvature $\mathrm{K}_{\mathrm{G}}=0$. Indeed a cylinder can be cut and unrolled to a planar state applying a pure bending deformation. On the contrary, this does not apply to a sphere. Recalling that bending deformations produces zero average in-plane strains of the surface, it now becomes clear that Gaussian curvature is related to the in-plane strains of the surface. In particular, $\mathrm{K}_{\mathrm{G}}$ identifies a surface's metric, which is the way we calculate distances and areas on it. To grasp the concept of a surface's metric we refer to the following experiment. We draw a circle of radius $r$ on a sphere, a plane or

\footnotetext{
8 Gambhir 2004.
}

9 Pressley 2009. 
a saddle-shaped surface (which have respectively $\mathrm{K}_{\mathrm{G}}>0, \mathrm{~K}_{\mathrm{G}}=0, \mathrm{~K}_{\mathrm{G}}<0$ ). We do this with the help of a thread (of length $\mathrm{r}$ ), attaching one extremity to a point on the surface and the other one to a pen, taking care of keeping the thread in contact with the surface while tracing the circle. The length of the resulting circle will be $2 \pi \mathrm{r}$ on the plane, but lower and higher in the sphere and the saddle, respectively.

The condition of $\mathrm{K}_{\mathrm{G}}=0$ identifies a special class of surfaces called developable surfaces, that are flat up to a bending deformation. The developability condition $\left(\mathrm{K}_{\mathrm{G}}=0\right)$ implies that one of the two principal curvatures is zero (recalling that $\mathrm{K}_{\mathrm{G}}$ is defined as their product). We already have seen that a cylinder is a developable surface: in this case the direction of zero curvature is the same everywhere besides cylinders there are two more types of developable surfaces: cones and the so-called tangent developables. In each point of a cone, the zero curvature direction points to the cone's vertex. Tangent developables are the surfaces built by "extruding" a curve in the three-dimensional space along its tangent. Therefore, they are locally flat along the extrusion direction and hence developable.

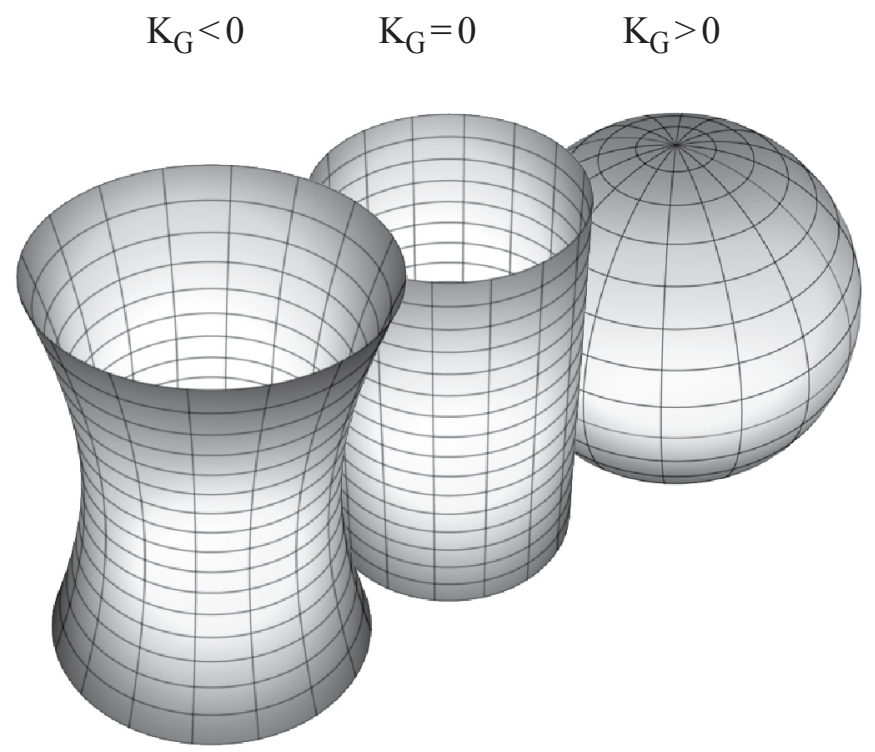

Fig. 2: A saddle, cylinder and sphere, respectively examples of surfaces with negative, zero and positive Gaussian curvatures $\left(\mathrm{K}_{\mathrm{G}}\right)$.

From our standpoint, of studying surfaces that change shape, the Gaussian curvature has a fundamental relevance. Surfaces with same K are called isometric and (as we have seen for cylinder and plane) can be transformed to one another keeping the distances unchanged (this is the main result of Gauss' Theorema Egregium ${ }^{10}$ ). In general (just like what we

10 Gauss 1902. 
have seen for the compressed bar) surfaces preferentially undergo some isometric shape change when subjected to a load (as we have seen thin bodies are much less rigid when bent than stretched). For developable surfaces $\left(\mathrm{K}_{\mathrm{G}}=0\right)$ such isometric transformation is easy to predict because it involves only bending. Instead, for non-developable surfaces, bending is always coupled to stretching: in this case the transformation is not isometric and therefore the shapes that can be acquired will depend strongly on the relative amount of bending and stretching energies involved, and buckling events (switching from one mode to the other) can appear when these two energy contributions have similar magnitude. Therefore, we will describe the shape change of thin bodies in terms of both the in-plane strains and the curvature-changes of their mid-surface.

\subsection{Fundamentals of spontaneous shape change}

\subsubsection{Generating forces and deformations: eigenstrain and eigencurvature.}

Besides external mechanical loading, shape transformations can occur also due to other types of interaction of the body's material with the environment. For example, when we soak water beads ${ }^{11}$ the hydrogel material of which the beads are made absorbs large amounts of water, and the beads increase in volume, undergoing large strains (identified by a zero at the subscript: $\varepsilon_{0}$, see also fig. 3a). We define this "non-mechanical" strain as the eigenstrain: it is the spontaneous or natural strain of a material which is sensitive to changes of the environment, such as temperature, light, humidity, electric or magnetic field. This character is well conveyed by the german word eigen which means own. The strain that we introduced previously is now called elastic strain, to highlight its mechanical origin. When both a mechanical and environmental stimuli are applied, for instance if we squeeze the swollen gel bead, the elastic strain is the difference between the total strain (this is the "visible" one that can be measured as described for the rubber sheet) and the eigenstrain; in other terms, the elastic strain is defined with respect to the swollen configuration that acts as a reference.

Even when only eigenstrains are present, residual stresses can develop and therefore elastic strains emerge. A typical example (fig. 3b) is a thin plate made of two identical layers except for different thermal expansion properties (one expands by $\varepsilon_{0}$ for a given temperature increase, the other one does not expand at all). As we could expect intuitively, when the temperature increases, the material cannot reach the local eigenstrain value because each layer constrains the other one. The expanding layer experiences a residual compressive stress while the passive layer a tensional one, being on average zero over the thickness. ${ }^{12}$ As a result, the plate bends in order to fulfill the different expansions.

11 These are used to irrigate plants over longer time, for instance during short holiday trips.

12 This scenario is not always granted: some eigenstrain distributions can also prevent the plate to possess a stress-free shape (on this point, see section 2.3.1). 


$$
\varepsilon_{0}=0
$$$$
\varepsilon_{0}>0
$$

$$
\kappa_{0}=0
$$$$
\kappa_{0}>0
$$

a

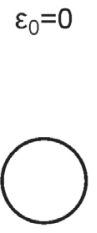

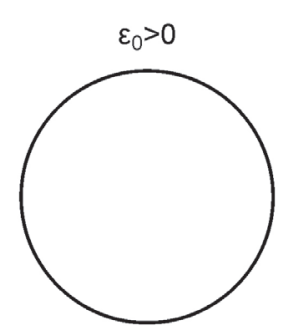

D

$$
\mathrm{K}_{0}=0
$$$$
\mathrm{K}_{0}>0
$$
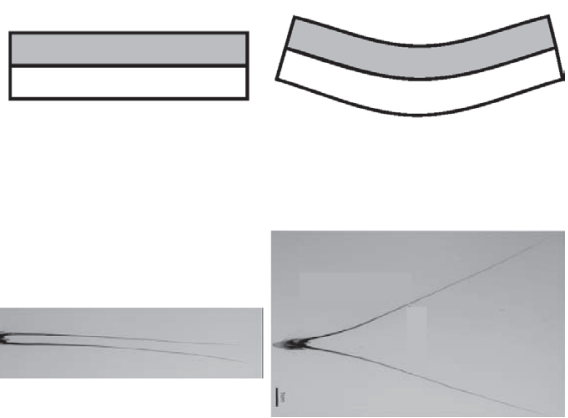

Fig. 3: Eigenstrain $\varepsilon_{0}$ and eigencurvature $\mathrm{k} 0$ are respectively spontaneous length and curvature changes. (a) Example of eigenstrain in a hydrogel: from the initial dry state $\left(\varepsilon_{0}=0\right)$ to the final swollen state $\left(\varepsilon_{0}>0\right)$ a large volume increase due to swelling of water takes place. (b) Example of eigencurvature in the awns of the wild wheat: each awn is a bilayer made of a hygroscopic (white) and non-expanding (grey) materials. Due to the humidity difference between night and day, the awns' shape changes from straight $\left(\kappa_{0}=0\right)$ to bent $\left(\kappa_{0}>0\right)$. Elbaum et al. 2007.

A natural bilayer has been described by Elbaum, ${ }^{13}$ in the awns of the wild wheat which foster the seed dispersal. Here, the bending is caused by differential expansion between the two layers that make up each awn due to changes in humidity between night and day. Clearly, both the artificial and the natural systems can be easily described in terms of eigencurvature, that is a natural curvature that develops in the awn in absence of external mechanical loading. It becomes now clear that eigenstrain (intended as a local change of lengths in the material) is fundamental for shape changing systems: in particular a non-uniform distribution of spontaneous expansion in a body (differential eigenstrains) as in the bilayer example are among the strategies implemented in many biological self-folding systems.

\subsubsection{Some strategies to generate controlled eigenstrains and eigencurvatures}

Analyzing the microstructure of biological materials can provide enough insight in the design strategies that have evolved in Nature to obtain shape changing materials. A fitting example for this is wood. When trees grow they produce new wood material. An idea of this process can be obtained by looking at the many rings in the cross section of a tree's $\log$ : the tree grows in diameter by adding layers of cells to the log. Therefore this growth process is one-way: unlike animals where tissues can be reabsorbed or remodeled, plants

13 Elbaum et al. 2007. 
lack this ability. This can be problematic because adding more material increases the weight that the tree has to sustain. Moreover additional external loading can emerge (like a condition of persistent wind). A solution is found through some specialized tissues that can expand or contract, in this way counteracting situations of unbalanced loads and securing the structural stability of trees. As explained by Fratzl et al. ${ }^{14}$ to understand the mechanism of expansion/contraction generation it is sufficient to consider the wood cell walls architecture and composition ${ }^{15}$ (fig. $4 \mathrm{a}-\mathrm{c}$ ). These cells have a prismatic shape with walls made of a swellable matrix reinforced by almost inextensible cellulose fibrils spiralling around the cells' main axis. Therefore swelling is possible only perpendicularly to the stiff non-swelling cellulose fibrils. By controlling the cellulose orientation (measured by the microfibril angle - MFA) during their development, plant cells can generate eigenstrains, and modulate them to tensile or compressive values. Such plant tissues are responsible not only for stress generation in trees but also in a variety of seed dispersal systems, such as the pine cone (fig. 4d) or the awns of the wild wheat (fig. 3b), that function in absence of any metabolic energy source. The energy needed to mechanically actuate and perform a shape change is harvested from the environmental changes in humidity - this is the reason why these systems are also called hygromorphs.

Another example of biologically developed eigenstrain is found in the seed capsule of a family of plants called Aizoaceae (commonly known as iceplants). They are remarkable because -unlike most hygromorphs where eigenstrains are typically in the $10 \%$ range-, their active tissue experiences a $400 \%$ eigenstrain as a result of water absorption. The origin of such large eigenstrains is found in the peculiar composition and architecture of the active tissue, as explained by Harrington ${ }^{16}$ and colleagues. At a microscopic scale it consists of a lignified honeycomb structure with diamond shaped cells whose walls are lined by a cellulose rich hygroscopic tissue (fig. 4e). As liquid water comes in contact with the tissue, the hygroscopic inner layer swells increasing its volume; the honeycomb structure directs this large volume increase in a large expansion along the cells' short axis.

Man-made materials are also subjected to eigenstrains. Hydrogels in particular are a special class of polymers undergoing large expansion (1000\% in volume) upon water sorption. Hydrogels have a chemical structure that can be described as a network of long chains connected together at points called cross-links. These chains are hydrophilic (water liking) therefore they "attract" water molecules when soaked. The degree of expansion depends on the density of cross-links: less cross-links bring to higher expansion. Moreover, they can be chemically synthesized to modulate the swelling in response to a variety of different stimuli (temperature, light, magnetic and electrical fields). Technological advances have made possible to fine tune both the stimuli-sensitivity (for instance one can control the

14 Fratzl/Elbaum/Burgert 2008.

15 Composition refers to the chemically different materials found in the tissue; architecture refers to the structure of the tissue at lower scales (microstructure, nanostructure, etc).

16 Harrington et al. 2011. 
temperature at which volume change occurs) and the spatial distribution of the cross-links in thin geometries (by lithographic techniques as shown, for example, by Klein and Kim, ${ }^{17}$ see fig. 4f). For these reasons, hydrogels have been the experimental system of choice to investigate the role of eigenstrains distribution onto the folding shape.

a

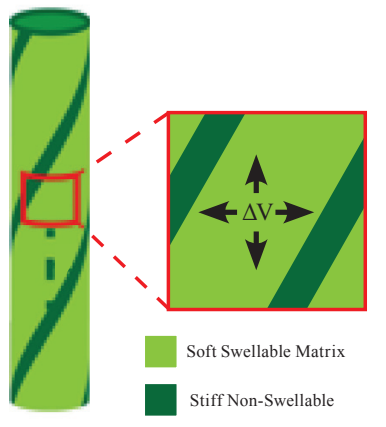

b

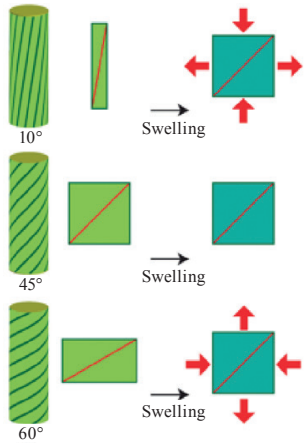

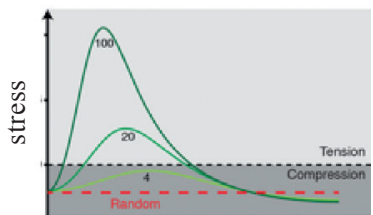

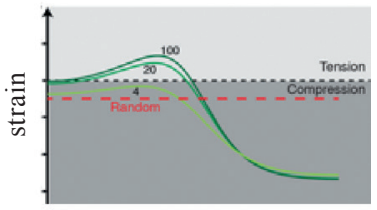

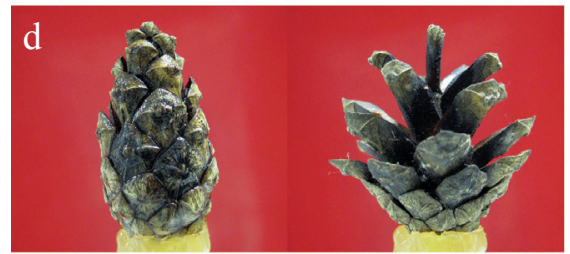
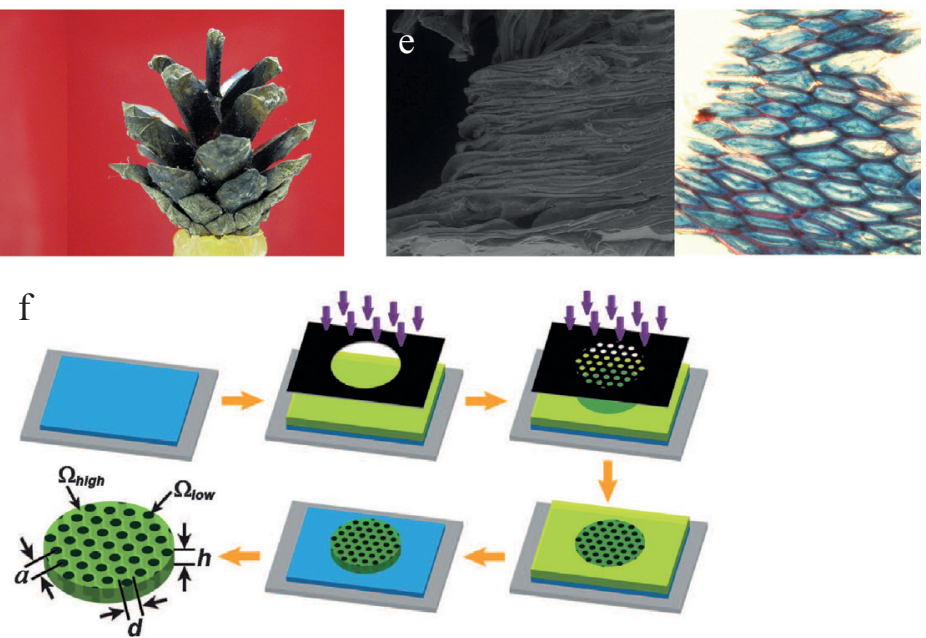

Fig. 4: Strategies to control eigenstrains in biological and artificial systems. (a-c) The cellulose microfibril angle (MFA) controls the mechanics and swelling of the plant cell wall. Dunlop 2016. (a) Schematic of a simplified cell wall, consisting of a soft swellable matrix reinforced by stiff fibres wrapped at an angle $\mu$ around the cell. (b) Schematic of how cells with different MFAs will respond to swelling of the soft matrix.

(c) Tensional and compressive strains and stresses can be generated depending on the MFA. (d) A pine cone, an example of actuating biological structures responding to change in environmental humidity based on the plant cell wall model. (e) Larger directed expansions are achieved in the active tissue of the iceplant seed capsule through a different spatial arrangement of the swelling (pale blue) and non-swelling (red) materials. (f) Schematic of halftone lithography: by changing the dimension of the low swelling black dots with respect to the high swelling green material fine spatial control of swelling properties in a hydrogel sheet is possible. Kim et al. 2012.

17 Klein/Efrati/Sharon 2007; Kim et al. 2012. 


\subsubsection{Minimal triggers for spontaneously morphing systems}

From what we have seen in this section, in order to achieve a shape change a self-folding system must possess two basic ingredients:

> ability to deform in response to a non-mechanical stimulus (eigenstrain)

> non-uniform distribution of eigenstrain across the body

Especially this second point is very relevant, as we will see, because the eigenstrain distribution influences which pattern of shape change arises. In reality the choice of this pattern or the evolution from one pattern to another is strictly subjected to the so-called boundary conditions. Mathematically speaking these are additional equations that have to be considered when searching for the equilibrium shape of a self-folding body. From a more practical perspective, these are physical constraints acting on the body: for instance, in the example of the spontaneously bending bilayer, boundary conditions describe how the extremities of the bilayer are treated (for example, if an extremity is glued to a support, and the other one is free to move along a carriage).

\section{Exploring the manifold of thin structures undergoing spontaneous folding}

\subsection{A classification of self-folding systems based on eigenstrains distribution}

To properly distinguish among the various types of biological and artificial self-folding systems, we choose to categorize them on the basis of the eigenstrain distribution and orientation. Moving along the vertical axis of fig. 5, a first distinction is made between systems that behave as monolayer or bilayer systems, that is systems that are respectively homogeneous or not across the thickness (first or second row of examples in the fig.). From what we have shown in section 1.2.1, this is equivalent to endow the surface with an eigenstrain or eigencurvature. A second distinction is made depending on the in-plane direction and distribution of the eigenstrain (moving along the horizontal axis): the eigenstrain can expand/contract the layer mostly along one direction (and be null on the perpendicular one) or all directions (isotropic); they can be uniform in the whole layer or be differently distributed in the layer (for instance concentrated at the edge). In the following, eigenstrain and eigencurvature will be expressed in their principal frame of reference as vectors $\varepsilon_{0}=\left[\begin{array}{ll}\Delta_{1} & \Delta_{2}\end{array}\right]$ and $\kappa_{0}=\left[\begin{array}{ll}\kappa_{1} & \kappa_{2}\end{array}\right]$. In this notation, the subscripts 1,2 refer to the principal directions of the sheet (both flat and curved), indicated by the axes 1, 2 in fig. 5 . 


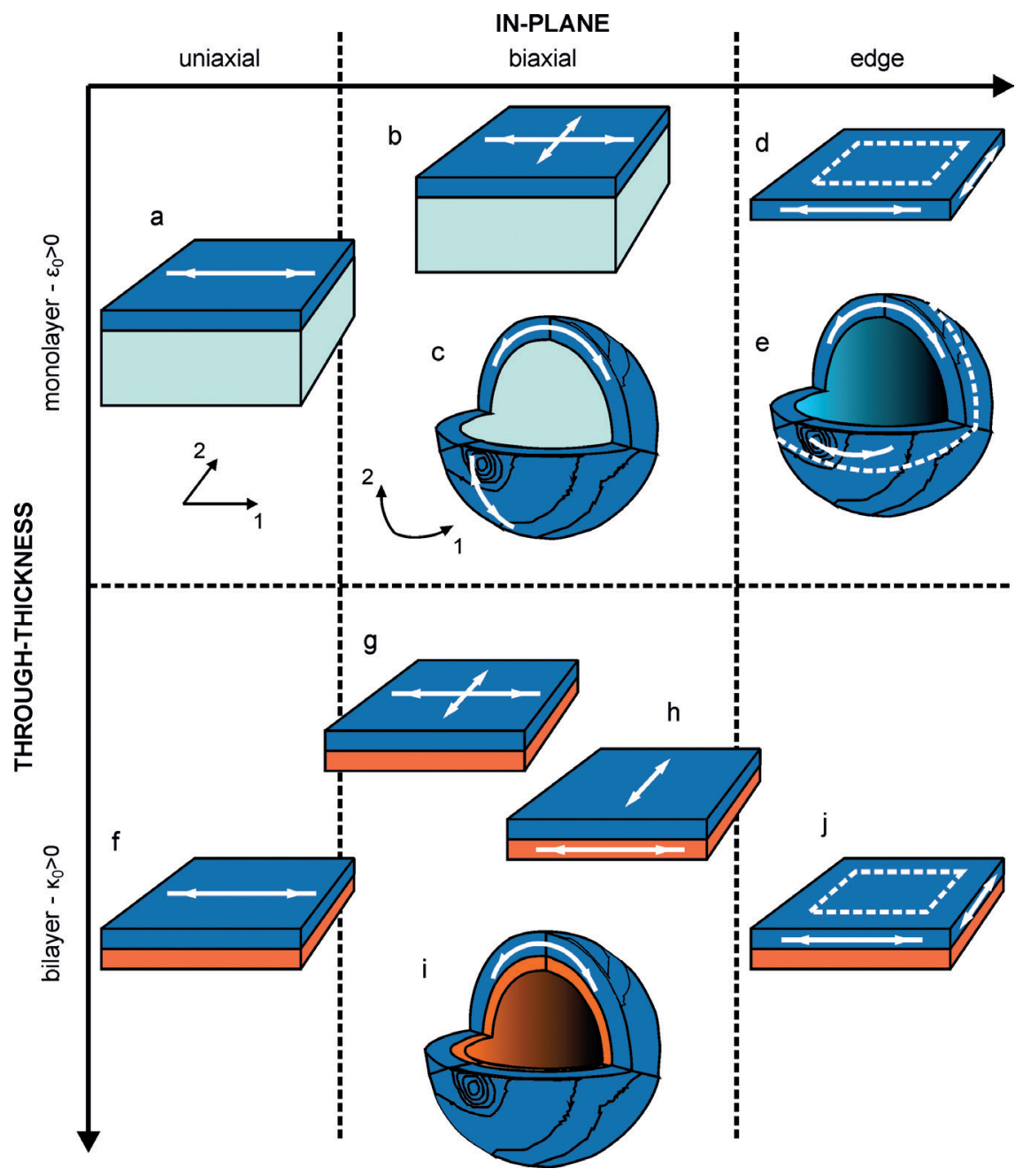

Fig. 5: Different types of self-folding systems triggered by in-plane (horizontal axis) and through-thickness (vertical axis) distribution of eigenstrains. Top row, from left to right: monolayer systems characterized by a uniaxial, biaxial and edge confined eigenstrains distribution. Bottom row, left to right: bilayer systems characterized by single, double and edge confined eigencurvature (eigencurvature derives from a through-thickness distribution of eigenstrains). The principal values of eigenstrains and eigencurvatures are directed along the white arrows. The geometry of the system (flat or doubly-curved) as well as the boundary conditions to which it is subjected (pale blue material schematizes a soft elastic foundation) have strong influence on the folding process. 


\subsection{Soft matter with hard skin}

In this section we present some examples of shape-changing systems formed by a thin and stiff sheet attached to a soft substrate (respectively schematized in fig. $5 \mathrm{a}-\mathrm{c}$ by dark blue and pale blue materials). In all these cases the shape change is due to a differential strain between these two portions: this gives rise to a manifold of different shapes and patterns.

\subsubsection{Shape changes in a sheet subjected to uniaxial confinement}

To start off we consider the shape transformation of a thin sheet that is attached to a soft substrate. This is a common schematization of many systems that appear at disparate length scales, both in biological and artificial systems, ${ }^{18}$ such as the shape of a polyester sheet resting on a liquid or a soft hydrogel ( $\mathrm{mm} \mathrm{scale}$ ), or tri-colloidal systems and even surfactant-water-air interface in the lungs alveola ( $\mathrm{nm}$ scale). All these systems start from a flat state that subsequently morphs into a wavy (wrinkled) configuration and eventually reach a convoluted, folded one. The morphing can be caused by a lateral compression of the sheet or, equivalently by a unidirectional expansion of the sheet subjected to a lateral confinement: both cases can be described by defining the normalized excess length of the sheet (fig. 5a). In mathematical formalism the sheet is subjected to an eigenstrain $\varepsilon_{0}=\left[\begin{array}{ll}\Delta & 0\end{array}\right]$ with $\Delta>0$ since the sheet expands with respect to the substrate. ${ }^{19}$ The progression of the shape change depends on the magnitude of $\Delta$ as we will show hereafter.

Ideally, compressing the sheet by $\Delta$ should shorten while keeping it flat. Instead, even for very minor compression, it starts wrinkling (fig. 6a). The wrinkling is the onset of a first mechanical instability which is in fact very similar to the buckling of an axially loaded slender bar: from our experience we know that it is very difficult to shorten the bar, which instead will preferentially deform by bending. The shape of the wrinkled sheet is a sine wave with a profile length $\lambda_{0}$, where $\lambda_{0}$ depends both on the bending rigidity of the sheet $B$ and the effective stiffness of the substrate $K$. This can be readily understand this if we first consider the sheet separately from the substrate. If we would compress the sheet alone, it would bend acquiring a single arched shape, pretty much like as for the axially loaded bar: this is because a single large arch has a smaller curvature than many smaller ones. On the other hand, a single arch would cause a large deformation of the substrate, since the latter is bonded to the sheet and follows its vertical displacement. Therefore the substrate switches to a configuration with many arches. Eventually the actual length of the sine profile $\lambda_{0}$ is a tradeoff between these two competing processes.

18 Pocivavsek et al. 2008; Genzer/Groenewold 2006; Brau et al. 2013.

19 With reference to fig. $5 \mathrm{a}$, considering a principal reference system aligned with the sheet sides, $\Delta$ is the eigenstrain component along the horizontal direction (marked by a white arrow), whereas the component along the perpendicular direction is zero. This notation is explained in section 2.1. 


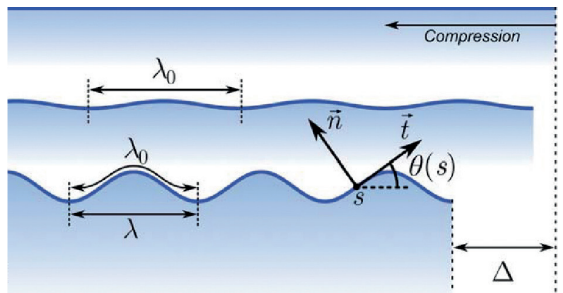

$\mathrm{b}$
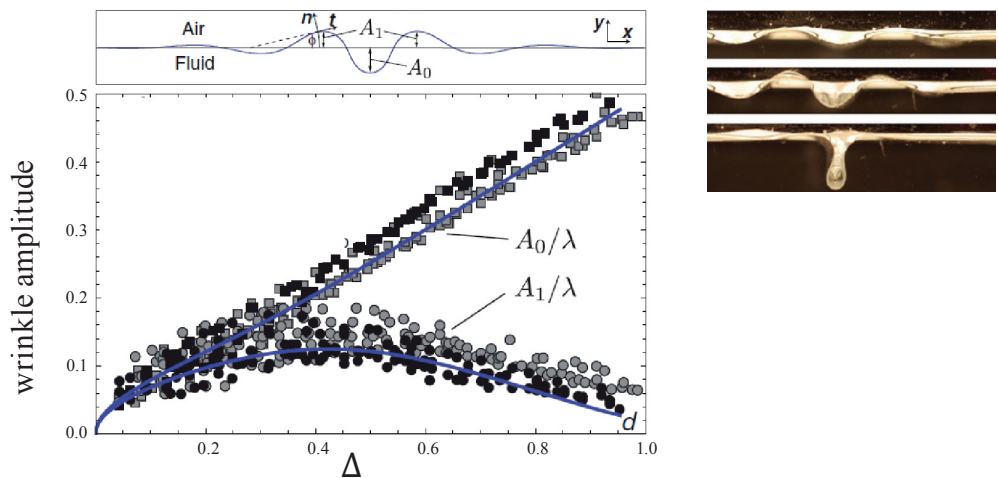

C
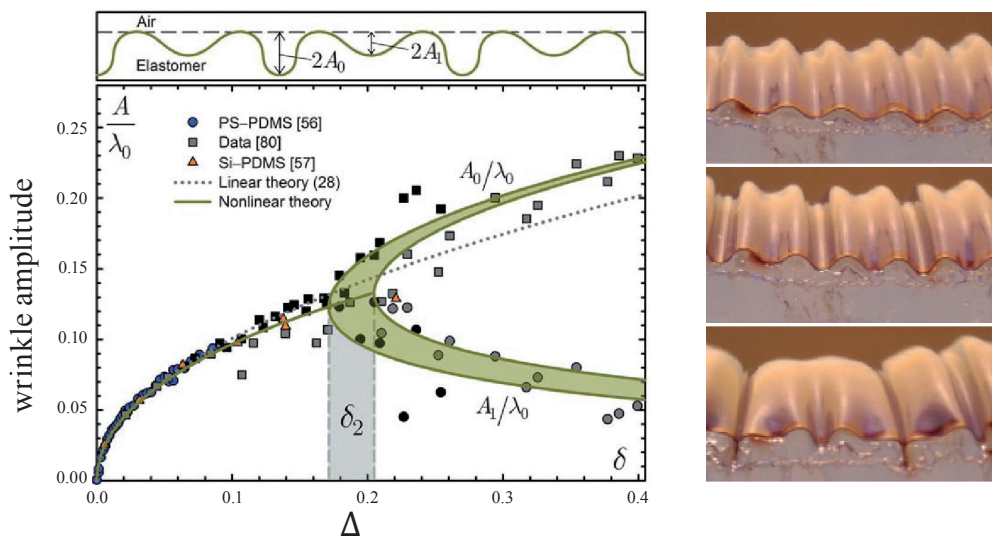

Fig. 6: Wrinkling and folding in a sheet subjected to uniaxial eigenstrain $\Delta$ with respect to an elastic foundation. (a) The initial straight state is lost in favor of a periodic wrinkled pattern. (b) Wrinkle-to-fold transition when the foundation is a liquid: past a certain threshold, the central fold amplitude $\mathrm{A}_{0}$ grows at the expense of flanking wrinkles $A_{1}$. (c) Wrinkle-to-fold transition when the foundation is a soft solid: flanking wrinkles do not disappear and the monolayer acquires a typical periodic profile with alternating deep folds and shallow wrinkles. Brau et al. 2013; Pocivavsek et al. 2008.

As $\Delta$ increases the wrinkled configuration is not stable anymore: this can be observed in the plot of fig. $6 \mathrm{~b}$, showing the measured amplitudes of the central wrinkle $\mathrm{A}_{0}$ and the first flanking one $\mathrm{A}_{1}$ taken from a plastic foil floating on a liquid. When $\Delta$ increases until about one third of the profile length $\lambda_{0}$, the wrinkled profile starts changing and the center wrinkle amplitude $A_{0}$ starts increasing linearly at the expense of the flanking ones. This bifurcation point is the onset of the wrinkle-to-fold transition. In the limit of larger and 
larger confinement, the center fold increases and all other wrinkles disappear. In reality this is not observed because the fold contacts itself when $\Delta=\lambda_{0}$

The reason why the fold appears depends on how the strain energy of the system scales with an increasing confinement $\Delta:^{20}$

$$
U \sim(B K)^{\square 2} \Delta-K \Delta^{3}
$$

the first term, with degree one half, is related to the winkling, and, as said, depends on both the sheet bending rigidity $B$ and substrate stiffness $K$; the second term with degree three is related to the folded state and depends only on $K$. When $\Delta$ is small the cubic term is negligible and the winkling solution is the most stable state. When $\Delta$ increases the cubic term lowers the total energy, favoring the folded state.

A slightly different folded configuration occurs when the substrate is a soft elastic material such as an elastomer (fig. 6c). Again in this case, when $\Delta$ is small the wrinkled shape is stable. ${ }^{21}$ Then, at a certain confinement $\delta_{2}$ the wrinkle-to-fold bifurcation is observed. In this case though, there are many deeper folds of amplitude $A_{0}$ flanked by smaller wrinkles of amplitude $\mathrm{A}_{1}$ which don't vanish even for very large $\Delta$. This behavior is due to the elastic response of the substrate: whereas in the sheet-fluid system the sheet could freely flow on the fluid and the central fold grow at the expense of the other wrinkles, here such a horizontal deformation of the substrate has a "cost" in terms of strain energy. As a result the folded configuration has a typical period-doubling shape (see fig. 6c) with alternating small amplitude wrinkles and large amplitude folds. If even larger confinement $\Delta$ is applied a period-quadrupling folding occurs: this suggests that a cascade of spatial period-doubling bifurcations could happen at high enough confinements (more than period-quadruple foldings however are hard to be observed experimentally because the sheet can undergo self-contact).

\subsubsection{Shape changes in a sheet subjected to biaxial confinement}

As an extension of the previous uniaxial strain states we now consider a biaxial compression or, equivalently a biaxial growth of the sheet. This scenario is a good approximation of what happens when our skin ages. Aging triggers biological modifications that make the skin superficial layer (epidermis) stiffer, while the internal layer tends to lose water causing its shrinking. This results in an excess length of the epidermis: mathematically the sheet is subjected to an in-plane eigenstrain $\varepsilon_{0}=\left[\begin{array}{ll}\Delta_{1} & \Delta_{2}\end{array}\right]$ with respect to the substrate (depicted by two perpendicular arrows in fig. 5b). We know from our daily lives that -from the unaesthetical orange peel appearance, to deep wrinkles- skin blemishes can be manifold.

20 Pocivavsek et al. 2008, 915.

21 The amplitude is proportional to the square root of $\Delta$ as with a fluid substrate. 
Moreover this problem has more than a merely aesthetical interest. Indeed a similar surface patterns appear in artificial material systems, for example depositing a thin metallic film ${ }^{22}$ onto an elastomer ${ }^{23}$ and letting the system cool down at room temperature, or by surface-modifying of polymers such as poly-dimetilsiloxane (PDMS) (see the examples described later).

In this case the buckling analysis has to account for all possible pairs of values $\Delta_{1}, \Delta_{2}$. Audoly and Boudaoud ${ }^{24}$ started studying this problem for values close to the critical $^{25}$ threshold value $\Delta^{\mathrm{C}}$. They showed that the sinusoidal wrinkled configuration (also called cylindrical) of frequency $k$ arising as the first buckled configuration in the monodimensional case is still a solution when the interfacial strain is small (below the critical value). For slightly larger eigenstrains $\Delta$ three additional possible patterns arise: the so-called, undulating, varicose and checkerboard patterns (fig. 7a). The undulating is obtained from straight wrinkles by lateral undulations of the crests and valleys. The varicose corresponds to a modulation of the amplitude of the straight wrinkles, along its crests and valleys. The checkerboard is obtained by superposition of two perpendicular sets of straight wrinkles. The hexagonal is obtained by superposition of three sets of straight wrinkles, at $2 \pi / 3$ angles.

These patterns are perturbed configurations of the cylindrical one, with respect to which a periodical perturbation of frequency $k$ ' along the longitudinal direction is superposed. Putting $q=k^{\prime} /{ }_{k}$ as the longitudinal wavevector relative to the cylindrical one, Audoly and Boudaoud present the stability of these patterns as a function of the two differential strains $\Delta_{1}, \Delta_{2}$ and their wavelength. In the phase diagram of fig. $7 \mathrm{~b}$ each portion of the plane is marked with the pattern that is more stable for the corresponding range of values of the two differential strains $\Delta_{1}, \Delta_{2}$. In particular they showed that the undulating pattern with large wavelength $(q=0)$ are more stable than the wrinkled state as soon as the strain compressing the wrinkling pattern longitudinally is higher than the critical value $\Delta_{2}>\Delta^{\mathrm{C}}$. The transition from wrinkled to varicose pattern is not observed because it takes place at strains that are much larger than for undulating stripes or for the checkerboard mode. When the differential strain is equibiaxial $\left(\Delta_{1}=\Delta_{2}\right)$, the checkerboard pattern is the more stable state above the critical thresholds, especially for larger $\Delta$. Remarkably, the equibiaxial case close to the initial buckling threshold $\left(\Delta_{1}=\Delta_{2}=\Delta^{\mathrm{C}}\right)$ is a special situation where the straight wrinkled state can coexist with all the other patterns. This is because all of the patterns (theoretically extrapolated or experimentally reported) can be seen as a superposition of straight wrinkles at different angles.

\footnotetext{
22 Here and throughout the rest of the text the term film is synonym of thin sheet.

23 Bowden et al. 1998.

24 Audoly/Boudaoud 2008a.

25 The critical threshold is the value of $\Delta_{1}, \Delta_{2}$ beyond which the system switches to a buckled state.
} 
a

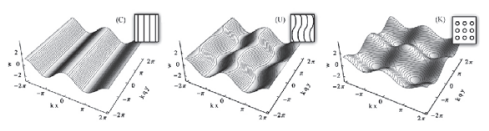

b

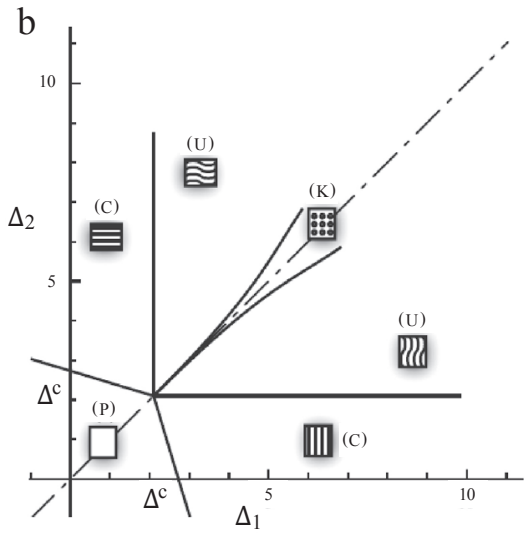

$\mathrm{C}$

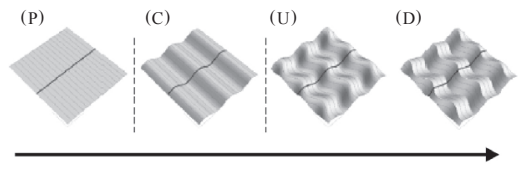

d

slow increase, trapping

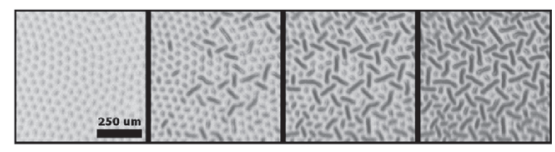

fast increase, no trapping

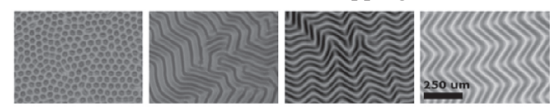

e

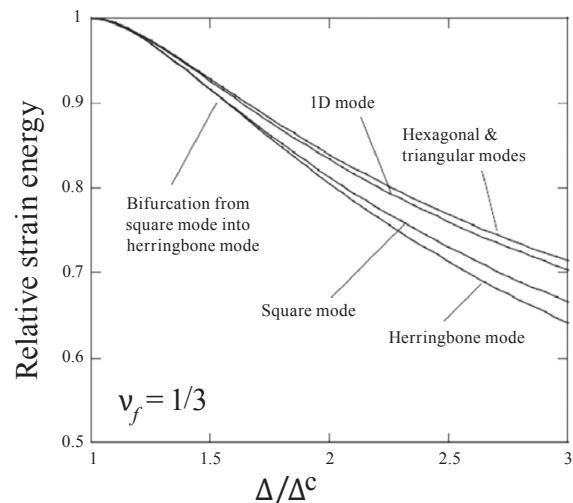

Fig. 7: Complex pattern formation and evolution in a monolayer subjected to increasing biaxial confinement resting on an elastic foundation. (a) Cylindrical, undulating and checkerboard patterns. (b) Phase diagram of the most stable patterns depending on the extent of eigenstrain along two perpendicular directions. Audoly/ Boudaoud 2008a. (c) Evolution of pattern in case of an increasing equibiaxial confinement: planar, cylindrical, undulating and developable patterns. Audoly/Boudaoud 2008b. (d) Influence of rate of change of confinement onto the pattern evolution: slow increase cause "trapping" from hexagonal pattern into disordered labyrinthine one; fast increase enable reaching the more stable herringbone pattern. (e) Relative strain energy of different patterns at increasing biaxial confinements: lower energies mean more stable patterns. Cai et al. 2011.

When the differential strain increases (see fig. 7c), the undulating pattern evolves smoothly (without a well-defined buckling threshold) towards a developable surface obtained by folding a cylindrical shape along sinusoidal ridges (pattern D in fig. 7c). This pattern (derived theoretically) is very close to the so-called herringbone (or zig-zag) pattern observed experimentally. In this pattern the spacing between the ridges along the transversal and longitudinal direction (with respect to the initial straight wrinkling) are comparable. By studying the energy of the developable pattern at very large differential strains, Audoly and Boudaoud showed that this configuration is far from the global minimum of energy. ${ }^{26}$

26 The global minimum is obtained when the spacing between ridges is much smaller than the wavelength of these sinusoidal ridges. 
This means that experimentally the system is trapped in a non-optimal state (herringbone pattern with comparable transversal and longitudinal wrinkling periodicity) although a more stable configuration could be possible. This so-called trapping mechanism depends on the history of loading of the system: if the differential strain beyond the secondary buckling threshold (from straight to undulated wrinkles) is increased slowly, the developable pattern inherits the wavelengths typical of the undulating pattern because this is a smooth change; if, instead, the change is fast, the system can reach the global minimum. The idea that loading history of the system has significance in the shape selection is also crucial in self-folding bilayered systems. Finally, at even larger $\Delta$ the developable pattern smoothly tends to a Miura-ori folding pattern. ${ }^{27}$ Again since this is a smooth transition trapping is possible.

Trapping in real experimental systems (as can be seen in fig. 7d) has an utmost importance because it constitutes a way to select different pattern under similar experimental conditions. For example, Cai and Hutchinson ${ }^{28}$ were able to show this in specimens of poly-dimethylsiloxane (PDMS) substrates that were treated with a chemical oxidative process that cause the stiffening of a thin superficial layer. These PDMS samples were then exposed to ethanol vapor: the ethanol would be absorbed first in the thin layer thus generating an equi-biaxial differential swelling. Moreover, by controlling the duration of exposure and the concentration of ethanol in the vapor, they were also able to control the speed and amount of differential swelling. Being able to inspect how the system would behave under a controlled equibiaxial differential strain $\varepsilon_{0}=[\Delta \Delta]$, they compared the pattern formation due to a large increase in $\Delta$, with slow and fast dynamics. With slow dynamics the system started from a pure hexagonal pattern, then isolated hexagons would coalesce with neighbors producing an extended local groove. Such groove formation was also triggering the coalescing of a neighboring pair into another groove that was not parallel to the original pair, in order to accommodate the local stress in an equi-biaxial manner. The overall result of such a sequence of individual buckling events was a pattern that locally resembles a segmented labyrinth, or a herringbone pattern with no global orientation (as observed by Huang and $\operatorname{Lin}^{29}$ ). In contrast, with a fast increase of domains of herringbone pattern were developed from the beginning and subsequently refined in extremely well-defined and larger domains.

To understand such a behavior, they derived upper bounds for the theoretical strain energy at low $\Delta$ of different patterns: the straight wrinkled, the checkerboard, hexagonal and herringbone. While close to the critical threshold all different patterns have similar energies (see fig. 7e), these become more separated at higher levels of differential eigenstrains.

27 Audoly/Boudaoud 2008c; a Miura-Ori folding pattern is a traditional folding in artistic origami, where the creases are zig-zag lines oriented as the crests and valleys of the developable pattern of fig. 7c.

28 Cai et al. 2011.

29 Huang/Hong/Suo 2005; Lin/Yang 2007. 
Their predictions clearly show that for higher $\Delta$ the more stable (lower energy) patterns were the checkerboard (square) and herringbone one followed by the straight wrinkled and hexagonal ones. While this result confirms the same conclusions drawn by Audoly and Boudaoud, it was striking that the theory could not explain the system's dominant preference for the hexagonal pattern when $\mathrm{D}$ was increased slowly. To explain this oddity the basic assumptions of the theory were reconsidered. In particular the authors questioned the assumption of a perfectly flat film. Indeed before any pattern formation, the films would show (even if very minor) an initial curvature. In fact, by introducing in the theory an outward curvature of the film, it could be shown that the hexagonal buckled mode is the more stable configuration (lower elastic energy minimum) in presence of a positive spherical curvature of the film. Moreover the curvature introduces a clear asymmetry in the buckling response: this was confirmed by the evidence that the hexagonal patterns on PDMS always showed inwardly oriented dimples. ${ }^{30}$

\subsubsection{Shape changes in a curved sheet subjected to equibiaxial confinement}

These results show how important the surface curvature is in the selection of a specific pattern. Moving on from this point, many researchers focused on the effects of curvature both theoretically ${ }^{31}$ and experimentally ${ }^{32}$ when the curved sheet is subjected to an homogeneous in-plane eigenstrain $\varepsilon_{0}=[\Delta \Delta]$ (see fig. 5c). The main outcome of these studies is that the surface curvature acts as an additional parameter to control the pattern formation: large changes of the film curvature (at a given level of differential strain) can push the system to a different pattern, exactly as does the critical strain far above the critical value. From the phase diagram in fig. $8 \mathrm{a}$, it can be seen that the hexagonal pattern is stable when the differential strain $\Delta$ and/or the radius of curvature $\mathrm{R}$ are low. Increasing $\mathrm{R}$ (relatively to the shell thickness $\mathrm{h}$ ) or $\Delta$ produces a so-called labyrinthine pattern with domains of curved ridges and valleys. Intermediate values of these parameters produce configurations of stable coexistence of the aforementioned patterns. At a closer inspection, these patterns are not trivial. Intuitively we would expect that for a very large radius of curvature. ${ }^{33}$ The film assumes the herringbone pattern observed by Cao and Hutchinson instead of the labyrinthine one (see fig. 8a). But the planar periodicity of the herringbone pattern is incompatible with a spherical domain. Similarly, the hexagonal pattern observed at low radius is not perfect: some dimples have only 5 neighbouring dimples, demonstrating the existence of pentagonal topological defects (disclinations) as required by Euler's polyhedral theorem. ${ }^{34}$ The wrinkling at the surface of a drying pea is a perfect system to observe such interplay between pattern formation and surface curvature (fig. $8 \mathrm{~b}$ ). They have an almost spherical

\footnotetext{
30 A symmetric buckling would equally produce inward or outward dimples; instead the experiments showed only inwardly oriented dimples: in fig. $7 \mathrm{~d}$ the inward dimples have a darker shade of grey.

31 Cao et al. 2008; Li et al. 2011; Stoop et al. 2015.

32 Breid/Crosby 2013.

33 That is in the limit of a flat film.

34 From Euler polyhedral theorem in the sphere there must be 12 dimples with 5 neighbors.
} 
a

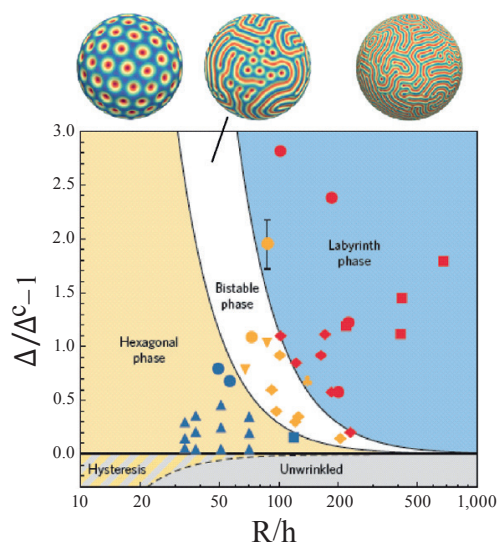

b

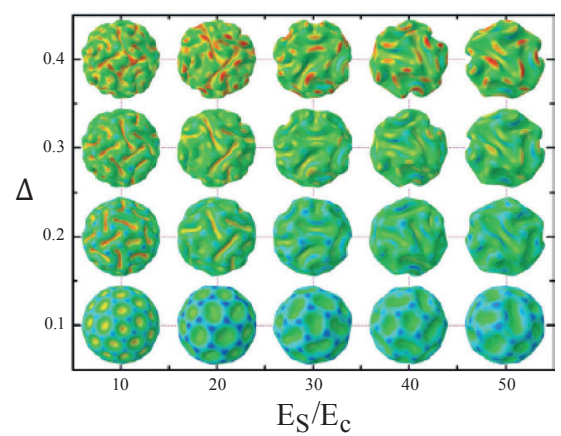

Fig. 8: Influence of curvature of the monolayer on the selection of buckling pattern. (a) Theoretical phase diagram and relative patterns (hexagonal, bistable and labyrinthine) as a function of surface curvature (normalized by monolayer thickness: $\mathrm{R} / \mathrm{h}$ ) and in-plane eigenstrains (beyond the onset of buckling: $\Delta / \Delta^{\mathrm{C}}-1$. Points correspond to real experiments on artificial system. Stoop et al. 2015. (b) Pattern formation on the skin of drying peas and relative numerical predictions as a function of skin-core modulus ratio and confinement. Li et al. 2011.

shape and, when drying, the softer inner core shrinks more rapidly than the stiff outer shell: also in this case there is a positive differential strain $\Delta$ between shell and core. Typically, due to the high shell thickness and the high stiffness ratio between shell and core, the dimples that are formed are quite large compared to the pea size. Therefore when $\Delta$ increases past a critical threshold a typical buckyball pattern appears, which shows a clear arrangement of hexagonal-pentagonal dimples. At even larger $\Delta$ neighboring dimples coalesce together forming a groove, in a secondary wrinkle-to-fold bifurcation. In this case since the groove length is comparable with the sphere radius, a true labyrinthine pattern is unfavored and grooves show a scattered orientation.

\subsection{Torn plastic bags and growing leaves: shape changes due to edge growth}

In this section we present some examples of systems where the shape change is caused by a positive differential strain of the edge with respect to the core of the sheet (see fig. $5 \mathrm{~d}-\mathrm{e}$ ). As the reader could already understand from the title of this section, this scenario has a very broad relevance, since it is applicable to a variety of different systems, both biological and artificial. 


\subsubsection{Non-uniform in-plane eigenstrain distributions}

All the examples introduced until now show that surface curvature, eigenstrains magnitude and to a certain extent also speed of deformation are possible means to control the pattern of shape change. As we have seen, such patterns consist of out of plane deflections from the sheet initial configuration that can be considered large in comparison with the sheet thickness, but are small with respect to the sheet size. Another line of research investigates large shape changes in finite size sheets through a reasoned distribution of the in-plane eigenstrain. This forward problem (given a three-dimensional target shape, find the corresponding in-plane eigenstrain distribution) has been dealt with for ideal surfaces (with vanishing thickness): the field of map projections provides a number of well-established conformal mappings ${ }^{35}$ of three-dimensional shapes onto flat surfaces (for example it is possible to almost completely map a sphere to a flat surface with the exception of a point).

The reverse problem, from a geometrical perspective, is to find the three-dimensional shape of a thin body corresponding to a given distribution of in-plane eigenstrains. This problem (as realized by Efrati, Sharon and Kupferman ${ }^{36}$ ) is not trivial and has been solved analytically only under simplifying axisymmetry assumptions. ${ }^{37} \mathrm{~A}$ thin body loses its intrinsic geometry as a result of a generic growth process. For example, let us assume, for simplicity, the thin body is flat: the growth process leads to an in-plane eigenstrain distribution. If the elastic plate is capable of satisfying the prescribed metric, then there exists a stress-free configuration, which is unique. That is the prescribed metric transforms the flat body to a curved one. If this is not possible, a residual in-plane stress will arise in the deformed plate, regardless of the assumed shape. Efrati and coauthors called such bodies non-Euclidean to highlight their impossibility to obtain a stress-free shape. Here non-Euclidean means that their internal geometry (prescribed by the metric) cannot be described only in terms of distances between points since such a pure geometrical description would overlook the presence of residual stress. In mathematical terms it is said that such bodies are not immersible in the three-dimensional Euclidean space, or, alternatively, the prescribed metric has no embedding in the Euclidean space.

\subsubsection{Edge growth in a flat sheet}

To explain such reverse problem, we once again resolve to a hand-held experiment, where we take a piece of plastic bag (flat in its resting configuration) and tear it apart (fig. 9a). A travelling crack is formed: the material flows perpendicularly to the crack as a result of the large extensional stresses. These material deformations close to the newly formed edges are not recovered after the load has been removed: the material has deformed plastically.

\footnotetext{
35 Ivanov/Trubetskov 1995.

36 Efrati/Sharon/Kupferman 2009a; Efrati/Sharon/Kupferman 2009b; Efrati/Sharon/Kupferman 2011; Efrati/Sharon/Kupferman 2013.

37 Dias/Hanna/Santangelo 2011.
} 
Hence we have prescribed a unidirectional eigenstrain along the edges. To be concise let us name this type of eigenstrain distribution as edge growth. The evident consequence of this can readily be seen: the edges have now assumed a rippled shape. Strikingly similar shapes are also observed in the shape of salad and kale leaves (fig. 9b): also in this case the edges grow more than the center of the leaf. The question is then: can we predict the edge shape corresponding to a given edge growth profile? Sharon ${ }^{38}$ et al. analyzed the shape of such torn plastic sheets and realized that the wrinkles at the sheet's edge are self-similar (or fractal): each bigger wave contains along its profile 3.2 smaller waves sharing the same amplitude-to-wavelength ratio, with wavenumbers according to a sequence:

$$
k, \alpha k, \alpha^{2} k, \alpha^{3} k, \ldots
$$

where $\alpha=3.2$ and $\mathrm{k}$ being the wavenumber of the first largest wave. Measuring the contour length of the sheet close to the edge, they could observe a contour excess length (with respect to the flat contour length) with a rapid decay moving from edge to center of the sheet (fig. 9c) which is mathematically equivalent to an eigenstrain $\varepsilon_{0}=[\Delta 0]$ aligned with the sheet's sides (see fig. 5d). That is, each narrow strip parallel to the edge is inplane compressed by the flanking one closer to the center. Recalling section 2.2.1, this is compatible with a straight wrinkling pattern with wavelength $\lambda$ (depending on the sheet stiffness and thickness). Therefore the cascade of waves is due to the superposition of many such wrinkling patterns with increasing wavenumber $\alpha^{\mathrm{i}} \mathrm{k}$ as one moves closer to the edge. Then the question arises: why is the cascade scaling factor about 3.2 ?

Audoly and Boudaoud ${ }^{39}$ showed that such period tripling waves are the most stable (lowest energy) types of wrinkling that can be achieved in a plate with a growing edge. To achieve this conclusion, he first realized that growth profile on the edge prescribes a given Gaussian curvature to the plate, on which the stretching energy depends. To minimize the elastic energy, the sheet assumes configurations that respect this prescribed curvature. In the limit of an infinitely thin plate, two families of embeddings (that is configurations respecting the growth profile) are possible, either with a unique wrinkling frequency or a cascade of superposed wrinkles. Because of the mathematical form of the elastic energy, its solutions must be invariant to a sign change. ${ }^{40}$ This means that the "self- similarity factor $\alpha$ must map odd integers to odd integers, [leaving] only odd integers $\left(3,5\right.$, etc.) as eligible values". ${ }^{41}$ Among these, fractal wrinkling patterns with $\alpha=3$ yields the most stable solutions and therefore are chosen.

\footnotetext{
38 Sharon et al. 2002.

39 Audoly/Boudaoud 2003.

40 The sign invariance mentioned here expresses the equivalence of two wrinkles which are specular with respect to the sheet plane.

41 Ibid, 3.
} 


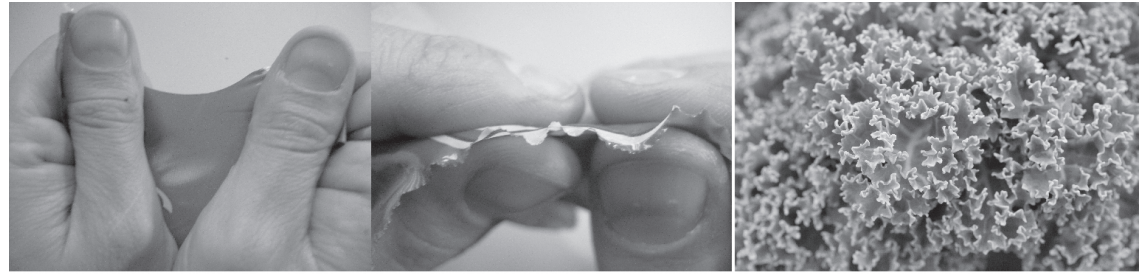

C

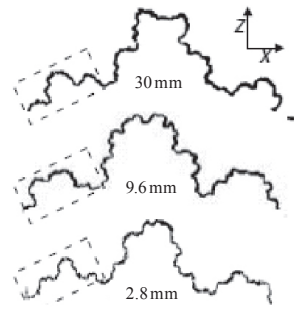
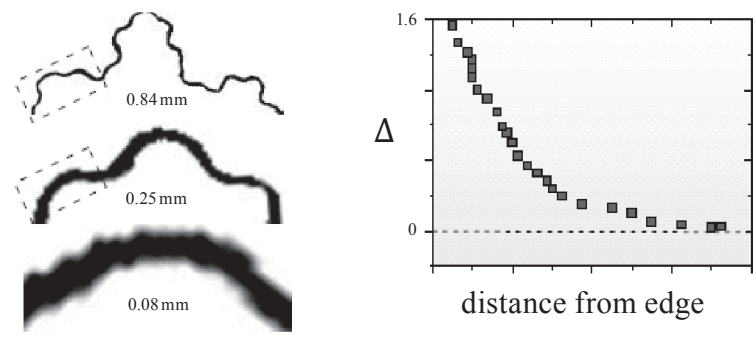

d
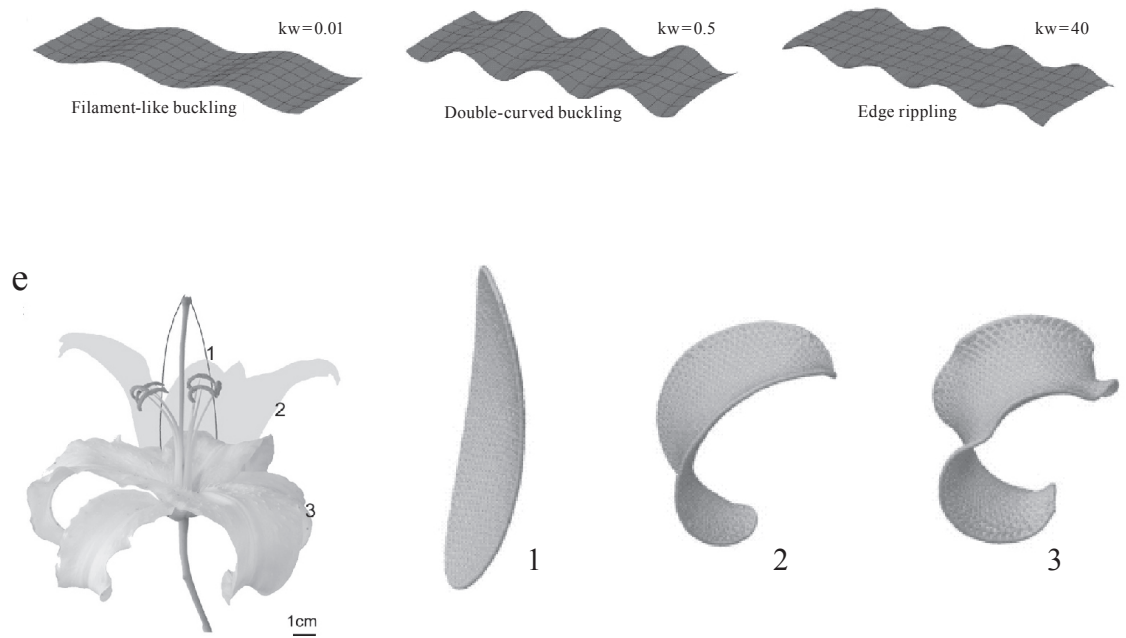

Fig. 9: Edge rippling induced by edge growth in monolayer systems. (a) When we tear a plastic bag, ripples arise at the newly formed edge which are strikingly similar to those observed in kale and salad leaves (b).

(c) Self-similar edge wrinkles in a torn plastic bag and measured growth strain. Sharon et al. 2002. (d) Influence of finite sheet size on the type of edge ripples in monolayers with "blade" geometry. Liang/Mahadevan 2009. (e) The blooming of a lily results from edge growth and its doubly curved geometry: in a first stage edge growth causes curvature inversion of the petal, that is blooming (1 to 2 ) and at a later stage ripple formation (2 to 3). Liang/Mahadevan 2011. 


\subsubsection{Edge growth in narrow flat sheets}

Liang and Mahadevan ${ }^{42}$ studied a special case of edge growth in a narrow plate, a scenario that is more similar to what happens in algal blades and lily leaves. ${ }^{43}$ Whereas in the previous examples of a torn plastic bag or a salad leaf the depth of the growth profile was much smaller than the sheet size, in these cases the finite width (w) of the plate has an influence on the type of shapes that can be achieved: algal blades can have both a saddle shape characterized by a negative Gaussian curvature everywhere or a flat configuration with rippled edges. To unravel the process of shape selection in growing blades, the authors calculated how bending and stretching energies scale in three possible configurations in presence of a large edge growth $\Delta$ confined close to the edge. The onset of a buckling mode happens when a critical growth $\Delta^{\mathrm{C}}$ is reached, where bending and stretching energies are in the same order of magnitude. Their analysis showed that three types of periodic buckling modes can arise depending on the ratio between the ripple wavelength $1 / \mathrm{k}$ (itself depending on the max value of $\Delta$ ) and the blade width w. In particular (fig. $9 \mathrm{~d}$ ), for $\mathrm{kw} \ll 1$ a one-dimensional buckling would occur where the blade wrinkles along the longitudinal direction and stays flat along the transversal one; for $\mathrm{kw} \sim 1$, the blade undergoes a doubly-curved buckling where the longitudinal wrinkling is accompanied by transversal curvature of opposite sign (a sequence of saddles); and finally an almost flat blade with an edge rippling of large wavenumber $\mathrm{k}$ is favored when $\mathrm{kw} \gg 1$.

\subsubsection{Edge growth in curved sheets}

The blooming process of a flower has similarities with the morphogenesis of leaves but it is worth considering since it sheds light on the role of initial shell curvature in the shape change process (fig. 5e). The traditional explanation is that the central midrib is responsible for the blooming of the lily: the leafy half of the midrib, growing more than the woody half, would generate the required outward bending. By excising the midrib from the petals when the lily is in the bud stage, Liang and Mahadevan ${ }^{44}$ observed that still an almost physiological blooming could occur. Moreover they observed ripples at the petal edges typical of edge growth, both in the bud and bloomed state. These evidences lead them to postulate an blooming mechanism driven by edge growth. In accordance to their measurements, they considered an edge growth $\Delta$ reaching farther towards the center of the petal (differently from the previous case of the "blade" leaves). Moreover, natural curved petals have a positive Gaussian curvature with a weak longitudinal curvature and strong lateral one, their ratio being $\mathrm{m}=\kappa_{\mathrm{x} 0} / \kappa_{\mathrm{y} 0} \in[0,1]$. This is a major departure from previous examples of edge growth in flat systems: as we have seen in section 1.1.5, in a surface with non-zero Gaussian

\footnotetext{
42 Liang/Mahadevan 2009.

43 Since only the shape of the plate changes, we still refer to an eigenstrain distribution as in fig. $5 \mathrm{~d}$.

44 Liang/Mahadevan 2011.
} 
curvature bending and stretching are coupled. As the growth strain increases from zero (fig. 9e), the shell unfolds slowly decreasing both curvatures. Past a certain critical value, the lower longitudinal curvature reverses, that is, the petal blooms. The theory shows that for the typical petal shape where $\kappa_{\mathrm{x} 0}$ is low (that is $\mathrm{m} \ll 1$ ), the curvature reversal is not sudden and the petal opens smoothly. Although no real buckling occurs (the blooming happens smoothly), the critical value has still a mechanical significance: it separates the stretching dominated and bending dominated regimes. Indeed after that value is passed, pure bending deformations in the form of edge-localized ripples arise.

\subsection{Systems with spontaneous curvature}

In this section we will give an overview of thin bodies that change shape as a result of a differential expansion across the thickness: this situation is equivalent to consider them as simple surfaces with a given natural (or eigen-) curvature and is schematized in the cases of fig. $5 \mathrm{f}-\mathrm{j}$.

\subsubsection{Developable sheets with a single eigencurvature}

In the most simple case, we consider a sheet which has at least one vanishing principal curvature, that is the sheet has a developable shape (as in fig. $5 \mathrm{f}$ where the sheet is flat). If an eigencurvature is prescribed, the sheet simply fulfills it through a bending deformation. This is the case, for instance, of the wild wheat awns (fig. 3b) and the pine cone in (fig. 4d) where the eigencurvature is caused by a differential hygroscopic expansion through the thickness. We will not pause longer on this class of morphing since no other shape changes occur when the eigencurvature increases. Indeed, since the sheet is developable, bending and stretching modes are decoupled: therefore a larger eigencurvature will only result in larger bending of the sheet.

\subsubsection{Sheets with a non-developable initial or target shape e $^{45}$}

The situation gets more complicated if either the initial shape or target shape are not developable (see fig. $5 \mathrm{~g}-\mathrm{i}$ ). A typical example of the first type (non-developable initial shape, fig. 5i) is the manifold of shapes that cells can acquire (from spherical to elongated to biconcave shape typical of red blood cells as in fig. 9a). Cells' membranes are vesicles made of a bilayer of free flowing lipid molecules. This makes them quite similar to soap bubbles. On the other hand, the two monolayers do not exchange lipid molecules and therefore area differences between them can give rise to spontaneous curvature. These two aspects influence the kind of shape to be acquired. Firstly the surface tension which

45 The target shape is the shape that is obtained (if possible) by fulfilling both the eigenstrain and eigencurvature. For example, a non-developable target shape is the saddle, corresponding to prescription of equal and opposite principal eigencurvatures to an initially flat sheet. 
(similarly to soap bubbles) tends to minimize the membrane area for a given volume: this drives towards spheres. The second is the spontaneous curvature: the larger the spontaneous curvature is, the more the vesicle will tend to satisfy it. Therefore surface tension and spontaneous curvature lead in general to contrasting shapes. Typically, spheres tend to buckle into prolate (elongated) and oblate (discoid) ellipsoids: from these shapes Helfrich, ${ }^{46}$ Seifert and Lipowsky ${ }^{47}$ together with Svetina and Zeks ${ }^{48}$ studied the types of possible evolutions as an increasing spontaneous curvature is ascribed. The shapes that were found could be placed in a geometric phase diagram with axes $v$ relative volume and $c_{0}$ relative spontaneous curvature (with respect to a sphere of same area). Already fixing a value of $c_{0}=0$ one can retrieve many shapes that are observed in real cells: starting from a sphere $(v=1)$ as the internal volume is decreased, prolate ellipsoids transition to dumbell shapes. Than a discontinuous transition leads to biconcave symmetric shapes similar to those observed in the red blood cells. At even smaller volumes, simply concave shapes (like stomatocytes) are the most stable. If one allows spontaneous curvatures to range from negative to positive values, even more complex features can be explained. In particular, prolate shapes dominate for $c_{0}>2$. At high values of $c_{0}$ symmetric dumbbell configurations become unstable and pear-shaped, asymmetric ones appear. Approaching the limit line $L^{\text {pear }}$ (the curved solid line at the top of fig. 10a) the dumbbells obtain narrow necks, which are loci of high and equal principal curvatures with opposite signs: past this limit line the neck collapses and the vesicle undergoes a so called budding (the smaller vesicle is ejected from the bigger one). At negative spontaneous curvatures instead oblate shapes dominate and can even lead to the inclusion of spherical cavities in the vesicle for large negative $c_{0}$ values. A remarkable transition is observed for oblate vesicles with slightly positive $c_{0}$ that decrease in volume: the vesicle self-intersects (this happens crossing the lines $\mathrm{SI}^{\mathrm{ob}}, \mathrm{SI}^{\text {sto }}$ from right to left) and acquires a toroidal shape.

\footnotetext{
46 Helfrich 1973.

47 Seifert/Lipowsky 1990; Seifert/Berndl/Lipowsky 1991.

48 Svetina/Zeks 1983; Svetina/Zeks 1989.
} 

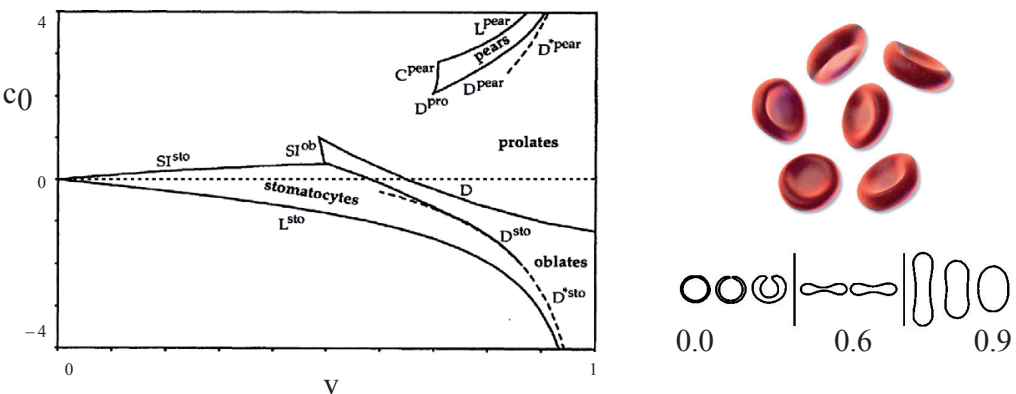

b
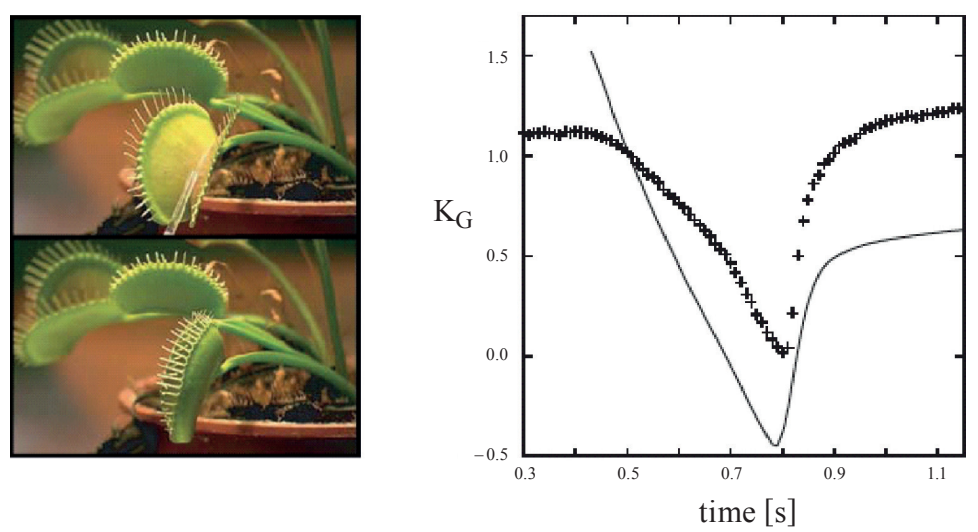

C
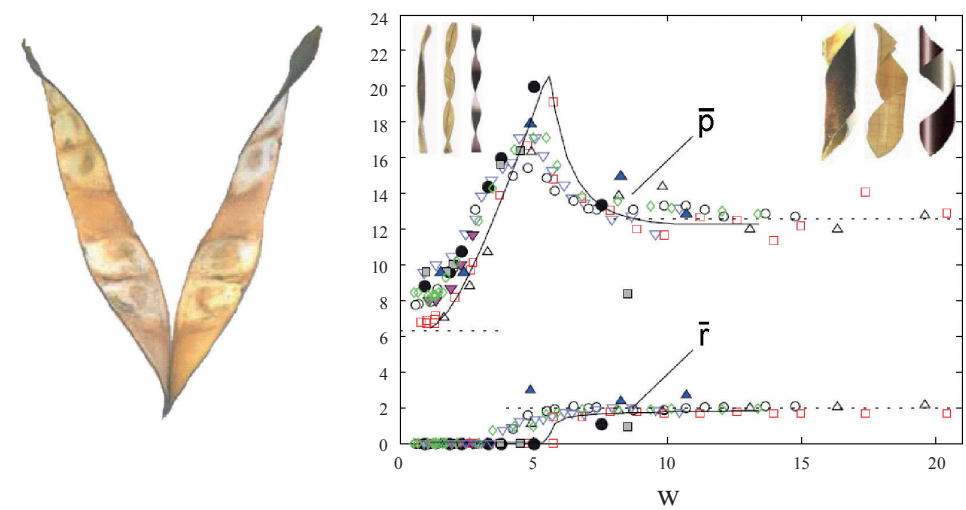

Fig. 10: Shape changes in systems endowed with a spontaneous curvature. (a) Phase diagram of cell shapes. Depending on the cell volume $\mathrm{v}$ and its spontaneous curvature $c_{0}$ a variety of shape changes is possible: prolate, oblate, concave, biconcave (like the red blood cells), toroidal cell shapes and even cell budding. Seifert/ Berndl/Lipowsky 1991. (b) Snapping closure of the Venus flytrap leaves is actively triggered by the plant (slow decrease of Gaussian curvature $\mathrm{K}_{\mathrm{G}}$ ) but the high speed of the motion (fast increase of $\mathrm{K}_{\mathrm{G}}$ ) is due to its doubly curved geometry (solid line, theoretical model; markers, experimental measurements). Forterre et al. 2005. (c) Chiral opening of the Bauhinia Variegata seed pods results from a competition between stretching and bending dominated behaviors, that eventually depend on the geometry of the pods (its width w) and non-developable target metric/curvature defined by the pod valves' material structure. The pitch $(\overline{\mathrm{p}})$ and radius $(\overline{\mathrm{r}})$ of the pod valve mark two possible shapes: a twisted helix or a ribbon (solid line, theoretical model; markers, experimental measurements). Armon et al. 2011. 
Another example of thin shell with non-developable initial shape (fig. 5i) is the plant known as Venus flytrap. This plant has typical doubly curved leaves that are convex in the open state and concave in the closed state. The morphing between these two states happens through a fast snap that is used to trap a prey. Measuring the leaf Gaussian curvature $\mathrm{K}_{\mathrm{G}}$ as a function of time. Forterre and Mahadevan ${ }^{49}$ realized that it was not constant during the closure (see fig. 10b): it would first decrease slowly (when the leaf is still convex) and then increase very rapidly (when the leaf becomes concave). As we have seen for the blooming lily, because the leaf is doubly-curved, the curvature inversion (convex-to-concave) can only happen through an intermediate state in which the leaf is stretched. To achieve this, the internal (turgor) pressure of the cells on the external side of the leaf is actively increased until the leaf is close to a critical curvature value. In this stage elastic energy is stored in the stretched leaf. As the turgor pressure is generated by a slower biochemical process $\mathrm{K}_{\mathrm{G}}$ decreases slowly. When a prey stimulates the plant "sensors" (cilia on the leaf inner face) only an additional small increase in turgor pressure is needed: the critical threshold is passed, the elastic energy released and the leaf switches to the more stable concave configuration. Moreover, since the Gaussian curvature $\mathrm{K}_{\mathrm{G}}$ is tipically higher than in the lily, the curvature inversion happens rapidly through a snap-through buckling. Therefore the overall mechanism ensures fast actuation, which is crucial for its prey-catching effectiveness.

The second scenario (non-developable target shape, fig. $5 \mathrm{~h}$ ) is well depicted by the seed pods of the plant Bauhinia variegatae: when the pod dries the two valves split open with a spiralling movement. Cues to understand this movement can be found already in the structure of the valves. As observed by Armon, ${ }^{50}$ each valve consist of two fibrous layers oriented roughly at $\pm 45^{\circ}$ with respect to the pod's longitudinal axis: therefore (recalling how wood actuation works, see section 1.2.2) the two layers shrink uniaxially at perpendicular directions. While each individual layer would stay flat, when they are attached together they develop opposite principal curvatures along the $\pm 45^{\circ}$ orientation of the fibers. With reference to fig. $5 \mathrm{~h}$ where the principal directions are along the sheet sides, the valve behaves as a sheet with target metric ${ }^{51} a_{0}=\left[\begin{array}{ll}0 & 0\end{array}\right]$ (where 0 means that no expansion/contraction is allowed due to the presence of fibers at perpendicular directions) and a target double curvature $b_{0}=\left[\kappa_{0}-\kappa_{0}\right]$. But such a case is special: the valve locally assumes a saddle-like configuration to satisfy the target curvature with a negative Gaussian curvature, the target metric requires null in-plane strains of the sheet; that is there is a metric incompatibility, where target metric and target curvature cannot be satisfied simultaneously. Therefore the strip will always have a residual stress. Such metric incompatibility is at the base of a strange behavior: despite having the same intrinsic curvature it was observed that wide strips adopt a ribbon shape (that is a cylindrical

\footnotetext{
49 Forterre et al. 2005.

50 Armon et al. 2011.

51 Target metric and target curvature are equivalent to eigenstrain and eigencurvature. The notation used here was given in section 2.1 .
} 
envelope shape) whereas narrow strips show a helical one (where the strip twists around its straight centerline). This can be deduced by the plot of fig. 10c where the experimentally measured pitch $(\overline{\mathrm{p}})$ is plotted versus radius $(\overline{\mathrm{r}})$ for many biological and artificial samples. Which configuration will be acquired depends on the minimum of the total energy. From energy scaling arguments Armon showed that when the strip width $w$ is large the system is stretching-dominated; this implies that to minimize the total energy one has to minimize the stretching energy, where the system satisfies the target metric $a=a_{0}$. Under this constraint the sheet assumes a curvature different from the target $b \neq b_{0}$, approximating it in one of the two principal directions while frustrating it (zeroing it) in the other: $b=\left[\begin{array}{ll}\kappa_{0} & 0\end{array}\right]$. This corresponds to the ribbon shape. Conversely, when the strip is narrow the sheet is bending dominated, and the energy is minimized for $b=b_{0}$. Under this constraint, $a$ has to approximate $a_{0}$. This corresponds to the helical configuration with twisted straight axis: at the midline the stretching energy is zero, but it increases with $w$. The switch from one regime to the other happens at a critical width $w_{c r} \approx 2.5 \sqrt{t / \kappa_{0}}$ where stretching and bending terms are comparable.

A similar case of sheets with a non-developable target shape, is observed in rectangular polymer-metal bilayers activated by an electrical-driven uniform contraction of the polymer layer (fig. 11a). Remarkably Alben ${ }^{52}$ observed that bilayers with a large length-to-width (aspect) ratio bend preferentially keeping the short side straight. Because the polymer contracts isotropically, the bilayer assumes a target spherical curvature $b_{0}=\left[\begin{array}{ll}\kappa_{0} & \kappa_{0}\end{array}\right]$ (fig. $5 \mathrm{~g}$ ). As we know, such a double curvature implies in-plane stretching, which is an unfavorable mode of deformation. When the polymer contracts, the bilayer first assumes a shallow spherical shape; afterwards it frustrates one of the two curvatures and bends along the other, assuming a cylindrical rolled shape. So why the short-side rolling ${ }^{53}$ would be always preferred? Alben realized that the bilayer would not bend into a perfect cylinder, but would preserve narrow regions of double-curvature close to the rolled edges. Since these regions are closer to the target curvature, they lowered the elastic energy of the system. Therefore the system prefers to roll on the short-sides thus maximizing the regions of double-curvature to the long sides.

\subsubsection{Sheets with a double eigencurvature confined at the edges}

This section on bilayers deals with the morphing of edge activated bilayers (fig. $5 \mathrm{j}$ ) where a spontaneous double curvature $b_{0}=\left[\begin{array}{ll}\kappa_{0} & \kappa_{0}\end{array}\right]$ appears at the edges. In a set of experiments on swelling polymer bilayers of variable rectangular shape, Stoychev ${ }^{54}$ observed a variety of rolling scenarios (fig. 11b): long side rolling in case of large aspect ratio bilayers; diagonal and all-side rolling in case of aspect ratios close to 1 . Strikingly enough, short side rolling was not observed at all: Stoychev's was in open contradiction with the experiments of

\footnotetext{
52 Alben/Balakrisnan/Smela 2011.

53 In short-side rolling the short side stays flat and the long ones bends.

54 Stoychev et al. 2012
} 
Alben. The main reason for the different behavior is due to the different activation. The polymer bilayers synthesized by Stoychev showed a swelling (active) layer attached to the substrate and a passive (non-swelling) layer on top. Since the active layer is confined between the substrate and the passive layer, water can diffuse in it only through the lateral faces (edges), and consequently swelling will occur first close to the edge and then reach more internal portions of the bilayer with time. Because swelling is confined to the edges, the bilayer does not transit through the shallow spherical shape. Therefore rolling starts at the corners first (where the diffusion depth is maximum) creating conical shapes; then two scenarios are possible. Long-side rolling is energetically favored for high aspect ratio because the costly in-plane stresses are relaxed (by bending) in a larger portion (the long edge). When instead the aspect ratio of the bilayer is about one, the bilayer can progress towards diagonal rolling (if rolling was initiated at two opposite corners) or all-side rolling (if it started at adjacent ones).

In a subsequent paper, Stoychev ${ }^{55}$ investigated the folding of polymer bilayers with more general shapes, such as ellipses or star shapes with a round contour (fig. 11c). In the early stages of swelling Stoychev observed the emergence of partially rolled ripples at the rounded edges, bestowing them with a characteristic "beer cap" contour. Moreover the number of ripples would decrease as the diffusion front travels deeper towards the center. Such edge rippling is in agreement with the edge growth in single sheets but, since the sheet is a bilayer, the edges preferentially bend in one out-of-plane direction, that is, the expected edge rippling will not be symmetric. At the same time it contradicts the formation of edge tubes observed in rectangular bilayers. In reality both processes occur, although at different time scales: the initial ripples would fuse together when the angle between them exceeds $130^{\circ}$ (this happens always for edges that are not too convex) forming the familiar tubular edge. Once long rolls are formed, they make the bilayer more rigid. As a result, after the edge tube formation bilayers can only fold over the lines connecting weak points between tubular edges. These movements resemble the folding operations in artistic origami, in which a flat sheet of paper is folded along specific lines to obtain a three-dimensional figure. It is remarkable that such a simple system characterized by isotropic mechanical and swelling properties undergoes complex multi-step actuation: again, the eventual folded shape depends from the previous stages acquired during the progression of the morphing process.

55 Stoychev et al. 2013. 
a

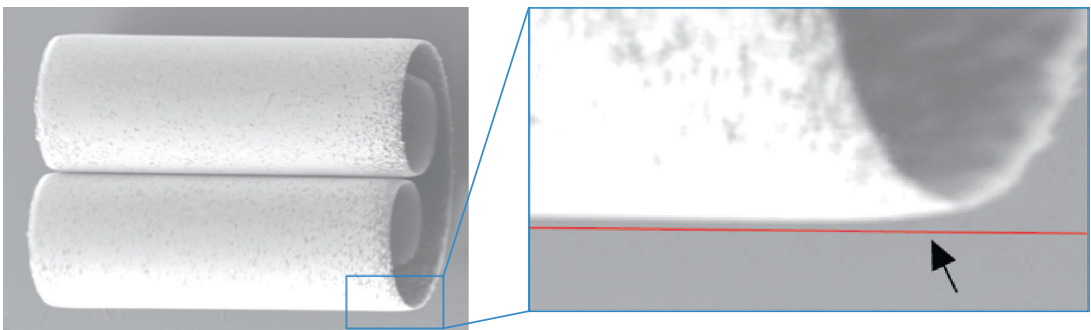

b
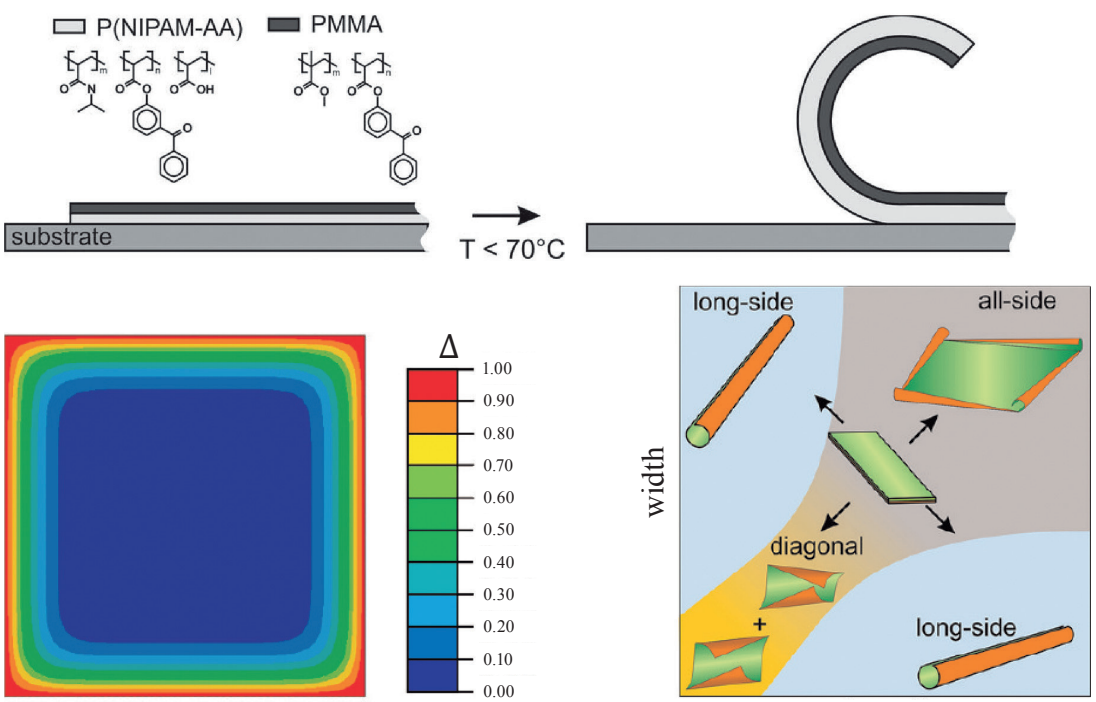

length

c
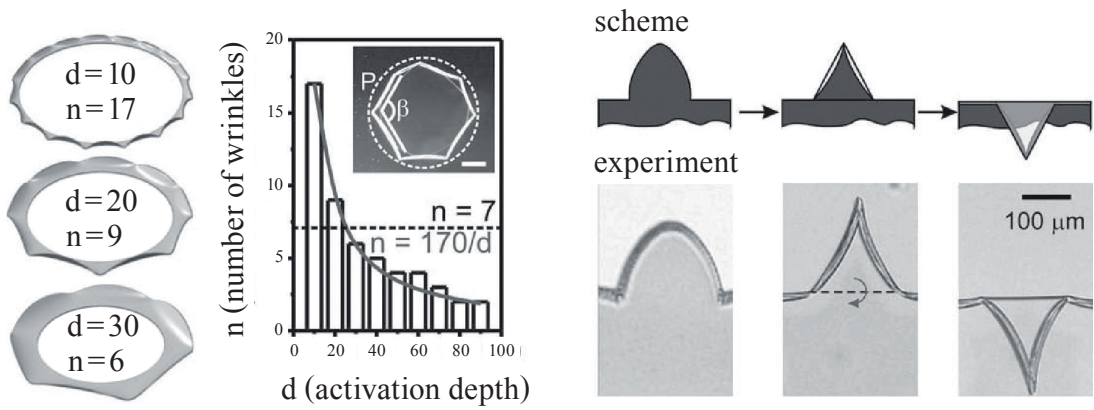

Fig. 11: Shape selection and evolution in bilayer systems with a spontaneous curvature subjected to edge effects. (a) Short side rolling is the preferred deformation mode in narrow bilayer strips in case of double eigencurvature: in this way the long edges maximize the extent of doubly curved edge portions. Alben/Balakrisnan/Smela 2011. (b) Scheme of bilayer composition (top), edge activation $\Delta$ (colored square) and phase diagram of rolling modes when the eigencurvature is activated at the edges first: in this case long side rolling is preferred because a doubly curved state of the edges is prevented from the initial adhesion to a substrate. Stoychev et al. 2012.

(c) Multistep asymmetric ripple formation and flap folding in swelling bilayers with complex star shapes: ripples form at the edges and (depending on their incident angles) fuse to rolls. When the edge is rolled the star arms are stiffened leaving only origami-like foldings as possible deformation. Stoychev et al. 2013. 


\subsection{Self-folding origami}

As a concluding remark we would like to at least mention a separate group of self-folding systems which is recently gaining more and more attention: the artificial self-folding origami. These systems are designed to experience extremely large eigenstrains and eigencurvatures at very narrow locations in the sheet: in fact a typical crease pattern appears, whereas the rest of the sheet is practically undeformed. This aspect makes such systems extremely convenient to be studied because their deformation depends only on the extent and time order of rigid body rotations at the creases. Bound to this is their success in robotic applications: to give an example, in a recent paper Felton ${ }^{56}$ demonstrated a prototype of robot that is able to fold itself from a flat creased plate, hold its own weight and even walk.

\section{Conclusion}

Although the present work is far from being exhaustive, we hope we have shed light on the underlying mechanisms that govern spontaneous shape change in thin systems. Despite the governing equations of plates subjected to eigenstrain being known since one century ${ }^{57}$, this branch of research is still quite active. This is due, in our opinion, to three main factors. First of all, these equations are scaleless: as we have seen in our examples many morphing processes could be explained regardless of their actual size, and therefore they can be applied to disparate systems. Second, the variety of patterns that can be predicted derives from the intrinsic nonlinearity of the elastic strain formulation ${ }^{58}$ : this makes the theory appealing to explain experimental observations in which little is known about the material properties (due to difficulties bound to the characterization methods or samples accessibility/availability). And third, especially relevant for theoretical research and simulation, is that there is a virtually infinite panorama of possible combinations of eigenstrains/eigencurvatures distributions, plate shapes and boundary conditions. In this perspective, the present paper is especially useful: presenting the folding of many different thin structures under the unifying concept of eigenstrain (and as consequence thereof), it enables to easily locate where research efforts have been focused until now. As a consequence, the paper calls for an exploration of less explored eigenstrain distributions: in turn, this will foster a broader and deeper understanding of self-folding systems and lead to new exciting engineering applications.

\footnotetext{
56 Felton et al. 2014.

57 Föppl 1907; von Kármán 1910.

58 The reader can verify that in all the examples the plate material has always been considered linear elastic.
} 


\section{Bibliography}

Alben, Silas/Balakrisnan, Bavani /Smela, Elisabeth (2011): Edge Effects Determine the Direction of Bilayer Bending. In: Nano Letters, vol. 11, no. 6, pp. 2280-2285.

Armon, Shahaf/Efrati, Efi/Kupferman, Raz/Sharon, Eran (2011): Geometry and Mechanics in the Opening of Chiral Seed Pods. In: Science, vol. 333, no. 6050, pp. 1726-1730.

Audoly, Basile/Boudaoud, Arezki (2003): Self-similar structures near boundaries in strained systems. In: Physical Review Letters, vol. 91, no. 8, 086105.

Audoly, Basile/Boudaoud, Arezki (2008a): Buckling of a stiff film bound to a compliant substrate - Part I: Formulation, linear stability of cylindrical patterns, secondary bifurcations. In: Journal of the Mechanics and Physics of Solids, vol. 56, no. 7, pp. 2401-2421.

Audoly, Basile/Boudaoud, Arezki (2008b): Buckling of a stiff film bound to a compliant substrate-Part II: A global scenario for the formation of herringbone pattern. In: Journal of the Mechanics and Physics of Solids, vol. 56, no. 7, pp. 2422-2443.

Audoly, Basile/Boudaoud, Arezki (2008c): Buckling of a stiff film bound to a compliant substrate - PartIII: Herringbone solutions at large buckling parameter. In: Journal of the Mechanics and Physics of Solids, vol.56, no. 7, pp. 2444-2458.

Audoly, Basile/Pomeau, Yves (2010): Elasticity and geometry: from hair curls to the nonlinear response of shells. Oxford: Oxford University Press.

Bowden, Ned/Brittain, Scott/Evans, Anthony G./Hutchinson, John W./Whitesides, George M. (1998): Spontaneous formation of ordered structures in thin films of metals supported on an elastomeric polymer. In: Nature, vol.393, no. 6681, pp. 146-149.

Brau, Fabian/Damman, Pascal/Diamant, Haim/Witten, Thomas (2013): Wrinkle to fold transition: influence of the substrate response. In: Soft Matter, vol.9, no. 34, pp. 8177-8186.

Breid, Derek/Crosby, Alfred J. (2013): Curvature-controlled wrinkle morphologies. In: Soft Matter, vol.9, no. 13, pp.3624-3630.

Cai, Shengqiang/Breid, D./Crosby, A. J./Suo, Zhigang/Hutchinson, John W. (2011): Periodic patterns and energy states of buckled films on compliant substrates. In: Journal of the Mechanics and Physics of Solids, vol.59, no. 5, pp. 1094-1114. 
Callister, William D. (2007): Materials science and engineering: an introduction. New York: John Wiley \& Sons.

Cao, Guoxin/Chen, Xi/Li, Chaorong/Li, Ailing/Cao, Zexian (2008): Self-assembled triangular and labyrinth buckling patterns of thin films on spherical substrates. In: Physical Review Letters, vol. 100, no. 3, 036102.

Deleuze, Gilles (1993): The fold: Leibniz and the Baroque. trans. Conley, Tom. London: The Athlone Press.

Dias, Marcelo A./Hanna, James A./Santangelo, Christian D. (2011): Programmed buckling by controlled lateral swelling in a thin elastic sheet. In: Physical Review E, vol. 84, no. 3, 036603 .

Dunlop, John W.C. (2016): The physics of shape changes in biology, Habilitation, Universität Potsdam.

Efrati, Efi/Sharon, Eran/Kupferman, Raz (2009a): Elastic theory of unconstrained non-Euclidean plates. In: Journal of the Mechanics and Physics of Solids, vol. 57, no. 4, pp. $762-775$.

Efrati, Efi/Sharon Eran/Kupferman, Raz (2009b): Buckling transition and boundary layer in non-Euclidean plates. In: Physical Review E, vol. 80, no. 1, 016602.

Efrati, Efi/Sharon Eran/Kupferman, Raz (2011): Hyperbolic non-Euclidean elastic strips and almost minimal surfaces. In: Physical Review E, vol. 83, no. 4, 046602.

Efrati, Efi/Sharon Eran/Kupferman, Raz (2013): The metric description of elasticity in residually stressed soft materials. In: Soft Matter, vol. 9, no. 34, pp. 8187-8197.

Elbaum, Rivka/Zaltzman, Liron/Burgert, Ingo/Fratzl, Peter (2007): The role of wheat awns in the seed dispersal unit. In: Science, vol. 316, no. 5826, pp. $884-886$.

Euler, Leonhard (1953): Opera Omnia. Zurich: Orell Füssli Verlag.

Felton, Samuel M./Tolley, Michael/Demaine, Erik/Rus, Daniela/Wood, Robert (2014): A method for building self-folding machines. In: Science, vol. 345, no. 6197, pp. 644-646. Föppl, August (1907): Vorlesungen über technische Mechanik. Leipzig: B. G. Teubner. 
Forterre, Yoel/Skotheim, Jan M./Dumais, Jacques/Mahadevan, Lakshminarayanan (2005):

How the Venus flytrap snaps. In: Nature, vol. 433, no. 7024, pp. 421-425.

Fratzl, Peter/Elbaum, Rivka/Burgert, Ingo (2008): Cellulose fibrils direct plant organ movements. In: Faraday Discussions, vol. 139, pp. 275-282.

Gambhir, Murari Lal (2004): Stability analysis and design of structures. Berlin, Heidelberg, New York: Springer-Verlag.

Gauss, Karl Friedrich (1902): General investigations of curved surfaces of 1827 and 1825. Princeton: The Princeton University Library.

Genzer, Jan/Groenewold, Jan (2006): Soft matter with hard skin: From skin wrinkles to templating and material characterization. In: Soft Matter, vol.2, no. 4, pp.310-323.

Harrington, Matthew J./Razghandi, Khashayar/Ditsch, Friedrich/Guiducci, Lorenzo/Rueggeberg, Markus/Dunlop, John W.C./Fratzl, Peter/Neinhuis, Christoph/Burgert, Ingo (2011): Origami-like unfolding of hydro-actuated ice plant seed capsules. In: Nature Communications, vol.2, no. 337, pp. 1-7.

Helfrich, Wolfgang (1973): Elastic Properties of Lipid Bilayers - Theory and Possible Experiments. In: A Journal of Biosciences: Zeitschrift für Naturforschung C, vol.28, no. 11, pp. $693-703$.

Huang, Zhiyong Y./Hong, Wei/Suo, Zhigang (2005): Nonlinear analyses of wrinkles in a film bonded to a compliant substrate. In: Journal of the Mechanics and Physics of Solids, vol. 53 , no. 9 , pp. $2101-2118$.

Ivanov, Valentin I./Trubetskov Michael K. (1995): Handbook of Conformal Mapping with Computer-Aided Visualization. Boca Raton: CRC Press.

Kim, Jungwook/Hanna, James A./Byun, Myunghwan/Santangelo, Christian D./Hayward, Ryan C. (2012): Designing Responsive Buckled Surfaces by Halftone Gel Lithography. In: Science, vol.335, no. 6073, pp. 1201-1205.

Klein, Yael/Efrati, Efi/Sharon, Eran (2007): Shaping of elastic sheets by prescription of non-Euclidean metrics. In: Science, vol.315, no. 5815, pp. 1116-1120.

Li, Bo/Jia, Fei/Cao, Yan-Ping/Feng, Xi-Qiao/Gao, Huajian (2011): Surface Wrinkling

Patterns on a Core-Shell Soft Sphere. In: Physical Review Letters, vol. 106, no. 23, 234301. 
Liang, Haiyi/Mahadevan, Lakshminarayanan (2009): The shape of a long leaf. In: Proceedings of the National Academy of Sciences of the United States of America, vol. 106, no. 52, pp. $22049-22054$.

Liang, Haiyi/Mahadevan, Lakshminarayanan (2011): Growth, geometry, and mechanics of a blooming lily. In: Proceedings of the National Academy of Sciences of the United States of America, vol. 108, no. 14, pp. 5516-5521.

Lin, Pei-Chun/Yang, Shu (2007): Spontaneous formation of one-dimensional ripples in transit to highly ordered two-dimensional herringbone structures through sequential and unequal biaxial mechanical stretching. In: Applied Physics Letters, vol.90, no. 24, 2419031.

von Kármán, Theodore (1910): Festigkeitsproblem im Maschinenbau. In: Enzyklopädie der mathematischen Wissenschaften, vol.IV, pp.311-385.

Pocivavsek, Luka/Dellsy, Robert/Kern, Andrew/Johnson, Sebastián/Lin, Binhua/Lee, Ka Yee C./Cerda, Enrique (2008): Stress and fold localization in thin elastic membranes. In: Science, vol.320, no. 5878, pp. $912-916$.

Pressley, Andrew (2009): Elementary differential geometry. London: Springer.

Seifert, Udo/Berndl, Karin/Lipowsky, Reinhard (1991): Shape Transformations of Vesicles - Phase-Diagram for Spontaneous-Curvature and Bilayer-Coupling Models. In: Physical Review A, vol.44, no. 2, pp. 1182-1202.

Seifert, Udo/Lipowsky, Reinhard (1990): Adhesion of Vesicles. In: Physical Review A, vol. 42 , no. 8 , pp. $4768-4771$.

Sharon, Eran/Roman, Benoît/Marder, Michael/Shin, Gyu-Seung/Swinney, Harry L. (2002): Mechanics: Buckling cascades in free sheets - Wavy leaves may not depend only on their genes to make their edges crinkle. In: Nature, vol.419, no. 6907, pp. 579-579.

Stoop, Norbert/Lagrange, Romain/Terwagne, Denis/

Reis, Pedro M./Dunkel, Jörn (2015): Curvature-induced symmetry breaking determines elastic surface patterns. In: Nature Materials, vol. 14, no. 3, pp. 337-342.

Stoychev, Georgi/Zakharchenko, Svetlana/Turcaud, Sebastien/Dunlop, John W.C./Ionov, Leonid (2012): Shape-Programmed Folding of Stimuli-Responsive Polymer Bilayers. In: ACS Nano, vol. 6, no. 5, pp. 3925-3934. 
Stoychev, Georgi/Turcaud, Sebastien/Dunlop, John W. C./Ionov, Leonid (2013): Hierarchical Multi-Step Folding of Polymer Bilayers. In: Advanced Functional Materials, vol.23, no. 18 , pp. $2295-2300$.

Svetina, Saša/Zeks, Boštjan (1983): Bilayer Couple Hypothesis of Red-Cell Shape Transformations and Osmotic Hemolysis. In: Biomedica Biochimica Acta, vol. 42, no. 11-12, pp. S86-S90.

Svetina, Saša/Zeks, Boštjan (1989): Membrane Bending Energy and Shape Determination of Phospholipid-Vesicles and Red Blood-Cells. In: European Biophysics Journal with Biophysics Letters, vol. 17, no.2, pp. 101-111.

Thompson, D’Arcy Wentworth (1953): On growth and form. Cambridge: University Press.

\section{Credits of Images}

Fig. 1, 5: (C) Photo: Lorenzo Guiducci, Berlin | Bild Wissen Gestaltung 2015.

Fig. 2: Adapted from the original, figure released in the public domain.

Fig. 3: (a), (b) above (C) Photo and Graphic: Lorenzo Guiducci, Berlin | Bild Wissen Gestaltung 2015; (b) below (C) Photo: Elbaum et al. 2007, fig. 4, adapted from the original, used with permission from The American Association for the Advancement of Science.

Fig. 4: (a -c) (C) Dunlop 2016, fig. 3-2; (d) Photo: John W. C. Dunlop, Potsdam | MPI für Kolloidund Grenzflächenforschung 2014; (e) Photo: Khashayar Razghandi, Potsdam | MPI für Kolloidund Grenzflächenforschung 2013; (f) C Kim et al. 2012, fig. 1, adapted from the original, used with permission from The American Association for the Advancement of Science.

Fig. 6: (a,c) (C) Brau et al. 2012, fig. 2, 7, adapted from the original, used with permission from The Royal Society of Chemistry; (b,c) (C) Pocivavsek et al. 2008, fig. 1, 2, adapted from the original, used with permission from The American Association for the Advancement of Science.

Fig. 7: (a,b) C Audoly/Boudaoud 2008a, fig. 2, 8, adapted from the original, used with permission from Elsevier; (c) (c) Audoly/Boudaoud 2008b, fig. 11, adapted from the original, used with permission from Elsevier; (d, e) (C) Cai et al. 2011, fig. 3, 4, 9, adapted from the original, used with permission from Elsevier. 
Fig. 8: (a) (C) Stoop et al. 2015, fig. 1, 3, adapted from the original, used with permission from Nature Publishing Group; (b) C Li et al. 2011, fig. 3, 5, adapted from the original, used with permission from American Physical Society.

Fig. 9: (a) Photo: Lorenzo Guiducci, Berlin | Bild Wissen Gestaltung 2015; (b) @ Photo released under license Creative Common 0, all rights are waived by the author; (c) (C) Sharon et al. 2002, fig. 1, adapted from the original, used with permission from Nature Publishing Group; (d) (C Liang/Mahadevan 2009, fig. 3, adapted from the original, used with permission from National Academy of Sciences of the United States of America; (e) (C) Liang/ Mahadevan 2011, fig. 1, 3, adapted from the original, used with permission from National Academy of Sciences of the United States of America.

Fig. 10: (a) (C) Seifert/Berndl/Lipowsky 1991, fig. 9, 10, adapted from the original, used with permission from American Physical Society; (C) Blausen Medical Communications, released under license Creative Common Attribution 3.0; (b) (C) Forterre et al. 2005, fig. 1, adapted from the original, used with permission from Nature Publishing Group; (c) Armon et al. 2011, fig. 1, 2, adapted from the original, used with permission from The American Association for the Advancement of Science.

Fig. 11: (a) (C) Alben/Balakrisnan/Smela 2011, fig.3, adapted from the original, used with permission from American Chemical Society; (b) Stoychev et al. 2012, fig. 1, p. 3925, adapted from the original, used with permission from American Chemical Society; (b, c) (C) Stoychev et al. 2013, fig. 2, 3, adapted from the original, used with permission from Advanced Functional Materials.

\footnotetext{
Lorenzo Guiducci

Email: lorenzo.guiducci@mpikg.mpg.de

Image Knowledge Gestaltung. An Interdisciplinary Laboratory.

Cluster of Excellence Humboldt-Universität zu Berlin.

Sophienstrasse 22a, 10178 Berlin, Germany.

Department of Biomaterials, Max Planck Institute of Colloids and Interfaces. 14476 Potsdam, Germany.
}

\section{John W.C. Dunlop; Peter Fratzl}

Department of Biomaterials, Max Planck Institute of Colloids and Interfaces. 14476 Potsdam, Germany.

This is the authors' version of the work. The definitive version was published as "An Introduction into the Physics of Self-folding Thin Structures” in: Friedman, Michael/Schäffner, Wolfgang (eds.): On Folding. Towards a New Field of Interdisciplinary Research in 2016 by transcript Verlag. The text is posted here by permission of transcript Verlag for personal use only, not for redistribution. 